\title{
A New Weibull Class of Distributions: Theory, Characterizations and Applications
}

\author{
Haitham M. Yousof ${ }^{*}$, , Mahbubul Majumder ${ }^{\ddagger}$, S. M. A. Jahanshahi*, \\ M. Masoom Ali ${ }^{\dagger \dagger}$ and G. G. Hamedani ${ }^{\dagger \dagger}$ \\ ${ }^{\dagger}$ Benha University \\ $\ddagger$ University of Nebraska \\ * University of Sistan and Baluchestan \\ ${ }^{\dagger \dagger}$ Ball State University \\ \$ Marquette University \\ Received: 6/25/2017 Approved: 8/29/2018
}

\begin{abstract}
We propose a new class of continuous models called the Weibull Generalized G family with two extra positive shape parameters, which extends several well-known models. We obtain some of its mathematical properties including ordinary and incomplete moments, generating function, order statistics, probability weighted moments, entropies, residual, and reversed residual life functions. Characterizations based on a ratio of two truncated moments, in terms of hazard function and based on certain functions of the random variable are presented. We estimate the model parameters by the maximum likelihood method. We assess the performance of the maximum likelihood estimators in terms of biases and mean squared errors by means of two simulation studies. The usefulness of the proposed models is illustrated via three real data sets.
\end{abstract}

Keywords. Weibull model; characterizations; order statistics; maximum likelihood estimation; quantile function; generating function; moments.

MSC 2010: 47N30; 97K70; 97K80.

\footnotetext{
* Corresponding author

Copyright $(2)$ 2019. ASP Ins. This open-access article is published under the terms of the Creative Commons AttributionNonCommercial 4.0 International License which permits Share (copy and redistribute the material in any medium or format) and Adapt (remix, transform, and build upon the material) under the Attribution-NonCommercial terms.
} 


\section{Introduction}

Several classes of distributions have been proposed in the statistical literature by adding one or more parameters to generate new distributions. Some wellknown generators are Marshall-Olkin-G family by Marshall and Olkin (1997) and Gupta et al. (1998) who proposed the exponentiated-G class. Other generators that can be cited are Barreto-Souza and Simas (2013), Alzaatreh et al. (2013), Bourguignon et al. (2014), Yousof et al. (2015), Tahir et al (2016), Afify et al. (2016b), Afify et al. (2016a), Yousof et al. (2016), Merovci et al. (2016), Korkmaz and Genc (2016), Alizadeh et al. (2016), Afify et al. (2017), Hamedani et al. (2017), Cordeiro et al. (2017), Alizadeh et al. (2017a,b) Nofal et al. (2017), Yousof et al. (2017a,b), Brito et al. (2017), Korkmaz et al. (2017), Cordeiro et al. (2018), Hamedani et al. (2018), Korkmaz et al. (2018a,b), Yousof et al. (2018a,b), and Alizadeh et al. (2018), among others.

Let $g(x ; \xi)$ and $G(x ; \xi)$ denote the density and cumulative distribution functions (CDF) of the baseline model with parameter vector $\xi$. Then the CDF of the Weibull Generalized-G (WG-G) is defined by

$$
\begin{aligned}
F(x) & =F_{\beta, \theta, \xi}(x)=\beta \int_{0}^{\frac{1-\bar{G}(x, \xi)^{\theta}}{\bar{G}(x, \xi)^{\theta}}} t^{\beta-1} \exp \left(-t^{\beta}\right) d t \\
& =1-\exp \left\{-\left[\frac{1-\bar{G}(x, \xi)^{\theta}}{\bar{G}(x, \xi)^{\theta}}\right]^{\beta}\right\}, \quad x \in \mathbb{R} .
\end{aligned}
$$

Henceforth, $\bar{G}(x, \xi)=\bar{G}(x), G(x ; \xi)=G(x), f(x)=f_{\beta, \theta, \xi}(x)$ and $g(x ; \xi)=$ $g(x)$. The corresponding probability density function (PDF) is given by

$$
f(x)=\beta \theta g(x) \frac{\left[1-\bar{G}(x)^{\theta}\right]^{\beta-1}}{\bar{G}(x)^{\theta \beta+1}} \exp \left\{-\left[\frac{1-\bar{G}(x)^{\theta}}{\bar{G}(x)^{\theta}}\right]^{\beta}\right\},
$$

where, $x \in \mathbb{R}, \beta>0$ and $\theta>0$ are two additional shape parameters. The additional parameters induced by the new Weibull generator are thought as a means to furnish a more flexible distribution. In this paper, we study the WG-G family and give a comprehensive description of its mathematical 
properties. In fact, the WG-G family is motivated by its important flexibility in applications. By means of two applications, it is noted that the WG-G class provides better fits than at least ten other families each having the same number of parameters. The reliability function (RF) $R(x)$, hazard rate function (HRF) $\tau(x)$ and cumulative hazard rate function (CHRF) $r(x)$ of the random variable (r.v.) $X$ are given, respectively, by

$$
\begin{aligned}
& R(x)=\exp \left\{-\left[\frac{1-\bar{G}(x)^{\theta}}{\bar{G}(x)^{\theta}}\right]^{\beta}\right\}, \\
& \tau(x)=\frac{\beta \theta g(x)\left[1-\bar{G}(x)^{\theta}\right]^{\beta-1}}{\bar{G}(x)^{\theta+1}}
\end{aligned}
$$

and

$$
r(x)=\beta \theta g(x) \frac{\left[1-\bar{G}(x)^{\theta}\right]^{\beta-1} \exp \left\{-\left[\frac{1-\bar{G}(x)^{\theta}}{\bar{G}(x)^{\theta}}\right]^{\beta}\right\}}{\bar{G}(x)^{\theta \beta+1}\left(1-\exp \left\{-\left[\frac{1-\bar{G}(x)^{\theta}}{\bar{G}(x)^{\theta}}\right]^{\beta}\right\}\right)} .
$$

The CDF of the WG-G family can be expressed as

$$
F(x)=1-\sum_{k=0}^{\infty} d_{1+k} \Pi_{1+k}(x),
$$

where

$$
d_{1+k}=\sum_{i, j=0}^{\infty}\left[(-1)^{i+j+k} / i !\right]\left(\begin{array}{c}
i \beta \\
j
\end{array}\right)\left(\begin{array}{c}
\theta(j-i \beta) \\
k
\end{array}\right)
$$

and $\Pi_{\gamma}(x)$ is the CDF of the Exp-G family with power parameter $(\gamma)$. Upon differentiating (3), we obtain the same mixture representation for the PDF

$$
f_{\beta, \theta, \xi}(x)=\sum_{k=0}^{\infty} t_{1+k} \pi_{1+k}(x),
$$

where $t_{1+k}=-d_{1+k}$ and $\pi_{\gamma}(x)=\gamma G(x)^{\gamma-1} g(x)$. Equations (3) and (4) are the main results of this section. The basic motivations (justifications) for using the new ratio 


$$
\left[1-\bar{G}(x)^{\theta}\right] / \bar{G}(x)^{\theta}
$$

are the following: to produce a skewness for symmetrical models; to define special models with all types of the HRF; to construct heavy-tailed distributions for modeling various real data sets; to make the kurtosis more flexible compared to the baseline distribution; to generate distributions with left-skewed, right-skewed, symmetric, or reversed-J shape; to provide consistently better fits than other generated distributions with the same underlying model.

Suppose that we have a lifetime r.v., $X$, having a certain continuous $G$ distribution. The generalized ratio, $\left[1-\bar{G}(x, \xi)^{\theta}\right] / \bar{G}(x, \xi)^{\theta}$, that the probability of an individual (or component) following the lifetime $Z$ will die (fail) at time $t$ is $\left[1-\bar{G}(x)^{\theta}\right] / \bar{G}(x)^{\theta}$. Consider that the variability of this ratio of death is represented by the r.v. $X$ and assume that it follows the Weibull model with shape $\beta$. We can write

$$
\operatorname{Pr}(Z \leqslant x)=\operatorname{Pr}\left(X \leqslant \frac{1-\bar{G}(x)^{\theta}}{\bar{G}(x)^{\theta}}\right)=F_{\beta, \theta, \xi}(x)
$$

which is given by (1). The basic motivations (justifications) for generating a new distribution in practice are the following: to produce a skewness for symmetrical models; to define special models with all types of HRF; to construct heavy-tailed distributions for modeling various real data sets; to make the kurtosis more flexible compared to that of the baseline distribution; to generate distributions with left-skewed, right-skewed, symmetric, or reversed-J shape; and to provide consistently better fits than other generated distributions with the same underlying model.

This paper is organized as follows. Some special models are presented in Section 2. In Section 3, we derive some mathematical properties for the new family. Some useful characterizations are presented in Section 4. Maximum likelihood estimation for the model parameters is addressed in Section 5 as well as a simulation study to assess the performance of the estimators. In Section 6 , potentiality of the proposed models is illustrated by means of two real data sets. Section 7 ends with some concluding remarks. 


\section{Special Models}

\subsection{The WG Weibull (WGW) Model}

Consider the $\operatorname{CDF} G(x)=\left\{1-\exp \left[-(x)^{b}\right]\right\}$ of the Weibull $(\mathrm{W})$ distribution with shape parameter $b>0$. The PDF of the WGW model (for $x>0$ ) follows from (2). Some plots of the WGW PDF and HRF for the selected parameter values are displayed in Figure 1. Figure 1 reveals that the WGW density can be left skewed, right skewed and unimodal. The HRF of the WGW model can be increasing, decreasing and bathtub. The PDF of the WGW model can be written as

$$
f_{\beta, \theta, b}(x)=\beta \theta b x^{b-1} \frac{\left\{1-\exp \left[-\theta(x)^{b}\right]\right\}^{\beta-1}}{\exp \left[-\theta \beta(x)^{b}\right]} \exp \left(-\left\{\frac{1-\exp \left[-\theta(x)^{b}\right]}{\exp \left[-\theta(x)^{b}\right]}\right\}^{\beta}\right) .
$$
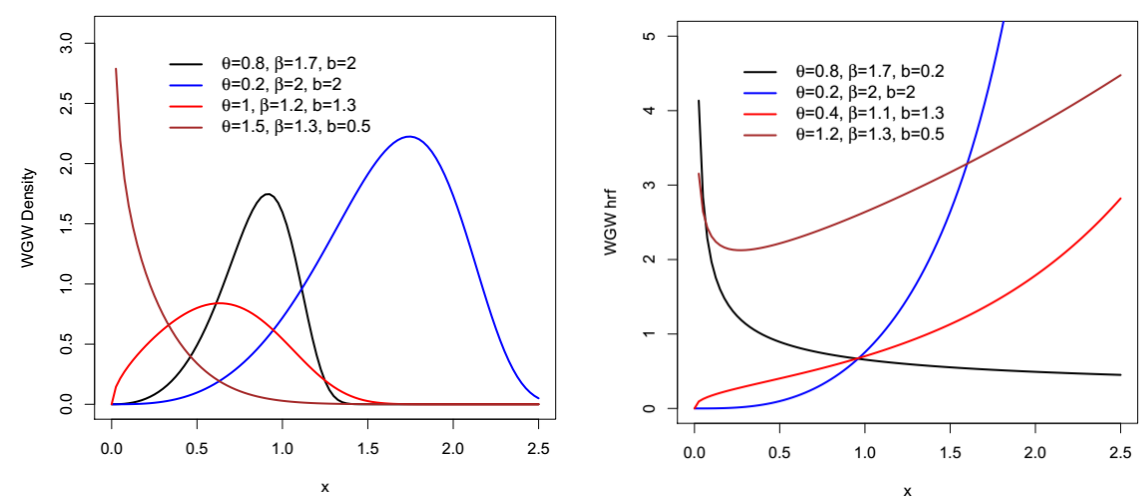

Figure 1. PDF (left) and HRF (right) plots of WGW distribution for selected parameter values.

\subsection{The WG Lindley (WGLi) Model}

Consider the CDF $G(x)=1-\frac{1+\alpha+\alpha x}{1+\alpha} \exp (-\alpha x)$ of the Lindley (Li) distribution with scale parameter $\alpha>0$. The WGLi density (for $x>0$ ) can be 
determined from (2) as

$$
\begin{aligned}
f_{\beta, \theta, \alpha}(x)= & \beta \theta \frac{\alpha^{2}}{1+\alpha}(1+x) \frac{\left\{1-\left[\frac{1+\alpha+\alpha x}{1+\alpha} \exp (-\alpha x)\right]^{\theta}\right\}^{\beta-1}}{\left.\left[\frac{1+\alpha+\alpha x}{1+\alpha} \exp (-\alpha x)\right)\right]^{\theta \beta}} \\
& \times \exp \left(-\left\{\frac{1-\left[\frac{1+\alpha+\alpha x}{1+\alpha} \exp (-\alpha x)\right]^{\theta}}{\left[\frac{1+\alpha+\alpha x}{1+\alpha} \exp (-\alpha x)\right]^{\theta}}\right\}\right) .
\end{aligned}
$$

Some plots of the WGLi density and hazard functions for selected parameter values are displayed in Figure 2. Figure 2 reveals that the WGLi density can be left skewed, right skewed and unimodal. The HRF of the WGLi model can be increasing and $\mathrm{J}$ shape.
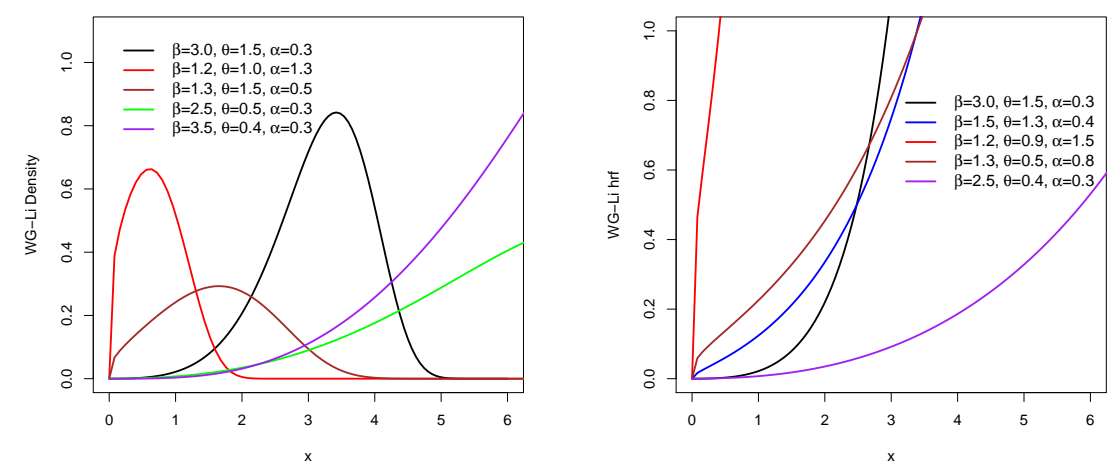

Figure 2. PDF (left) and HRF (right) plots of WGLi distribution for selected parameter values.

\section{Properties}

\subsection{Main Statistical Properties}

The $r$ th ordinary moment of $X$ is given by

$$
\mu_{r}^{\prime}=\mathbf{E}\left(X^{r}\right)=\int_{-\infty}^{\infty} x^{r} f(x) d x
$$


Then, we obtain

$$
\mu_{r}^{\prime}=\sum_{k=0}^{\infty} t_{1+k} \mathbf{E}\left(Y_{1+k}^{r}\right),
$$

where $Y_{\zeta}$ denotes a r.v. with the Exp-G distribution with power parameter $\zeta$. By setting $r=1$ in (5), we get the mean of $X$. The last integration can be computed numerically for most parent distributions. The skewness and kurtosis measures can be calculated from the ordinary moments using well-known relationships. The moment generating function (mgf) $M_{X}(t)=$ $\mathbf{E}\left(e^{t X}\right)$ of $X$ can be derived from equation (3) as

$$
M_{X}(t)=\sum_{k=0}^{\infty} t_{1+k} M_{1+k}(t),
$$

where $M_{1+k}(t)$ is the mgf of $Y_{1+k}$. Hence, $M_{X}(t)$ can be determined from the Exp-G moment generating function. The main applications of the $1^{\text {st }}$ incomplete moment refer to the mean deviations and the Bonferroni and Lorenz curves. These curves are very useful in economics, reliability, demography, insurance and medicine. The $r$ th incomplete moment, say $\mathbf{I}_{r}(t)$, of $X$ can be expressed from (4) as

$$
\mathbf{I}_{r}(t)=\int_{-\infty}^{t} x^{r} f(x) d x=\sum_{k=0}^{\infty} t_{1+k} \int_{-\infty}^{t} x^{r} \pi_{1+k}(x) d x .
$$

For the WGW model we have the following results (for any $r>-b$ )

$$
\mu_{r}^{\prime}=\Gamma\left(1+\frac{r}{b}\right) \sum_{k . m=0}^{\infty} a_{k, m}^{(1+k, r)},
$$

and

$$
\mathbf{I}_{r}(t)=\gamma\left(1+\frac{r}{b},\left(\frac{1}{t}\right)^{b}\right) \sum_{k . m=0}^{\infty} a_{k, m}^{(1+k, r)}
$$

where

$$
\begin{gathered}
a_{k, m}^{(1+k, r)}=t_{1+k} \omega_{m}^{(1+k, r)}, \\
\omega_{\zeta}^{(\tau, r)}=\left[\tau(-1)^{\zeta} /(\zeta+1)^{(r+b) / b}\right]\left(\begin{array}{c}
\tau-1 \\
\zeta
\end{array}\right)
\end{gathered}
$$

and 


$$
\gamma(a, x)=\int_{0}^{x} t^{a-1} \exp (-t) d t=\Gamma_{x}(a)=\sum_{n=0}^{\infty}\left\{(-1)^{n} x^{a+n} /[n !(a+n)]\right\},
$$

is the incomplete gamma function.

\subsection{Probability Weighted Moments (PWMs)}

The $(s, r)$ th PWM of $X$ following the WG-G family, say $\lambda_{s, r}$, is formally defined by

$$
\lambda_{s, r}=\mathbf{E}\left\{X^{s} F(X)^{r}\right\}=\int_{-\infty}^{\infty} x^{s} F(x)^{r} f(x) d x .
$$

The $(s, r)$ th PWM of $X$ can be expressed as

$$
\lambda_{s, r}=\sum_{k=0}^{\infty} c_{1+k} \mathbf{E}\left(Y_{1+k}^{s}\right) d x
$$

where

$$
\begin{aligned}
c_{1+k}= & \frac{\beta \theta(-1)^{k}}{1+k} \sum_{m, i, j=0}^{\infty} \frac{(-1)^{m+i+j}(m+1)^{i}}{m ! i !}(r)_{k} \\
& \times\left(\begin{array}{c}
\beta(i+1)-1 \\
j
\end{array}\right)\left(\begin{array}{c}
\theta[-\beta(i+1)+j]-1 \\
k
\end{array}\right),
\end{aligned}
$$

and

$$
(\tau)_{\zeta}=\tau(\tau-1) \ldots(1+\tau-\zeta)
$$

is the descending factorial and $\zeta$ is a positive integer. For the WGW model we have

$$
\lambda_{s, r}=\Gamma\left(1+\frac{r}{b}\right) \sum_{k, m=0}^{\infty} c_{k, m}^{(1+k, r)}, \quad \forall r>-b
$$

where

$$
c_{k, m}^{(1+k, r)}=c_{1+k} \omega_{m}^{(1+k, r)} .
$$




\subsection{Entropies}

The Rényi entropy of a r.v. $X$ represents a measure of variation of the uncertainty which is defined by

$$
p_{\delta}(X)=\frac{1}{1-\delta} \log \int_{-\infty}^{\infty} f(x)^{\delta} d x, \delta>\left.0\right|_{\delta \neq 1} .
$$

Using (2), we can write

$$
f(x)^{\delta}=\sum_{k=0}^{\infty} d_{1+k} g(x)^{\delta} G(x)^{1+k}
$$

where

$$
\begin{aligned}
d_{1+k}= & (-1)^{k}(\beta \theta)^{\delta} \sum_{i, j=0}^{\infty}\left[(-1)^{i+j+k} /\left(i ! \delta^{-i}\right)\right] \\
& \times\left(\begin{array}{c}
\beta(i+\delta)-\delta \\
j
\end{array}\right)\left(\begin{array}{c}
\theta[-\beta(i+\delta)+j]-\delta \\
k
\end{array}\right) .
\end{aligned}
$$

Then, the Rényi entropy of the WG-G family is given by

$$
p_{\delta}(X)=\frac{1}{1-\delta} \log \left[\sum_{k=0}^{\infty} d_{1+k} \int_{-\infty}^{\infty} G(x)^{1+k} g(x)^{\delta} d x\right] .
$$

The q-entropy, say $Q_{q}(X)$, can be obtained as

$$
Q_{q}(X)=\frac{1}{q-1} \log \left\{1-\left[\sum_{k=0}^{\infty} d_{1+k}^{*} \int_{-\infty}^{\infty} g(x)^{\delta} G(x)^{1+k} d x\right]\right\}
$$

where $q>0, q \neq 1$. The Shannon entropy of a r.v. $X$, say $S H(X)$, is defined by

$$
S H(X)=\mathbf{E}\{-[\log f(X)]\} .
$$

It is the special case of the Rényi entropy when $\delta \uparrow 1$. 


\subsection{Order Statistics}

Let $X_{1}, X_{2}, \ldots, X_{n}$ be a random sample from the WG-G family of distributions and let $X_{1: n}, \ldots, X_{n: n}$ be the corresponding order statistics. The PDF of $i$ th order statistic, say $X_{i: n}$, can be written as

$$
f_{i: n}(x)=\mathrm{B}^{-1}(i, n-i+1) f(x) \sum_{j=0}^{n-i}(-1)^{j}\left(\begin{array}{c}
n-i \\
j
\end{array}\right) F^{j+i-1}(x),
$$

where $B(\cdot, \cdot)$ is the beta function. Inserting (5) and (6) in equation (7) and using a power series expansion, we have

$$
F^{j+i-1}(x) f(x)=\sum_{k=0}^{\infty} p_{1+k} \pi_{1+k}(x),
$$

where

$$
\begin{aligned}
p_{1+k}= & {\left[\beta \theta(-1)^{k} /(1+k)\right] \sum_{m, l, w=0}^{\infty}\left[(-1)^{m+l+w}(j+i-1)_{k}(m+1)^{l} /(m ! l !)\right] } \\
& \times\left(\begin{array}{c}
\beta(i+1)-1 \\
w
\end{array}\right)\left(\begin{array}{c}
\theta[-\beta(l+1)+w]-1 \\
k
\end{array}\right),
\end{aligned}
$$

and the PDF of $X_{i: n}$ can be expressed as

$$
f_{i: n}(x)=\sum_{j=0}^{n-i}(-1)^{j} \mathrm{~B}^{-1}(i, n-i+1)\left(\begin{array}{c}
n-i \\
j
\end{array}\right) \sum_{k=0}^{\infty} p_{1+k} \pi_{1+k},
$$

i.e, the density function of the WG-G $i$ th order statistic is a mixture of Exp$\mathrm{G}$ densities. Based on the last equation, we note that the properties of $X_{i: n}$ follow from those of $Y_{1+k}$. For example, the $q$ th moments of $X_{i: n}$ can be expressed as

$$
\mathbf{E}\left(X_{i: n}^{q}\right)=\sum_{k=0}^{\infty} \sum_{j=0}^{n-i}(-1)^{j} \mathrm{~B}^{-1}(i, n-i+1)\left(\begin{array}{c}
n-i \\
j
\end{array}\right) p_{1+k} \mathbf{E}\left(Y_{1+k}^{q}\right)
$$


For the WGW model we have

$$
\mathbf{E}\left(X_{i: n}^{q}\right)=\Gamma\left(1+\frac{q}{b}\right) \sum_{k, m=0}^{\infty} \sum_{j=0}^{n-i} c_{k, m, j}^{(1+k, q)}, \quad \forall q>-b,
$$

where

$$
c_{k, m, j}^{(1+k, q)}=(-1)^{j} \mathrm{~B}^{-1}(i, n-i+1)\left(\begin{array}{c}
n-i \\
j
\end{array}\right) p_{1+k} \omega_{m}^{(1+k, q)} .
$$

\subsection{Moments of Residual Life and Reversed Residual Life}

The $n$th moment of the residual life, say

$$
\left.\tau_{n}(t)\right|_{(X>t)} ^{(n=1,2, \ldots)}=\mathbf{E}\left[(X-t)^{n}\right]
$$

uniquely determines $F(x)$. The $n$th moment of the residual life of $X$ is given by

$$
\left.\tau_{n}(t)\right|_{(X>t)} ^{(n=1,2, \ldots)}=[1-F(t)]^{-1} \int_{t}^{\infty}(x-t)^{n} d F(x) .
$$

Therefore

$$
\left.\tau_{n}(t)\right|_{(X>t)} ^{(n=1,2, \ldots)}=[1-F(t)]^{-1} \sum_{k=0}^{\infty} t_{1+k}^{(\tau)} \int_{t}^{\infty} x^{r} \pi_{1+k}(x),
$$

where

$$
t_{1+k}^{(\tau)}=t_{1+k} \sum_{r=0}^{n}(1-t)^{n} .
$$

The $n$th moment of the reversed residual life, say

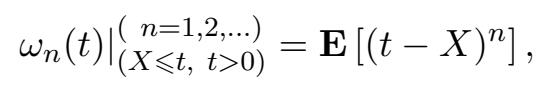

uniquely determines $F(x)$. We obtain

$$
\left.\omega_{n}(t) \omega_{n}(t)\right|_{(X \leqslant t, t>0)} ^{(n=1,2, \ldots)}=F^{-1}(t) \int_{0}^{t}(t-x)^{n} d F(x) .
$$

Then, the $n$th moment of the reversed residual life of $X$ becomes 


$$
\left.\omega_{n}(t)\right|_{(X \leqslant t, t>0)} ^{(n=1,2, \ldots)}=F^{-1}(t) \sum_{k=0}^{\infty} t_{1+k}^{(\omega)} \int_{0}^{t} x^{r} \pi_{1+k}(x),
$$

where

$$
t_{1+k}^{(\omega)}=t_{1+k} \sum_{r=0}^{n}(-1)^{r}\left(\begin{array}{l}
n \\
r
\end{array}\right) t^{n-r} .
$$

For the WGW model we have

$$
\begin{aligned}
\left.\tau_{n}(t)\right|_{(X>t)} ^{(n=1,2, \ldots)}= & {[1-F(t)]^{-1} \Gamma\left(1+\frac{n}{b},\left(\frac{1}{t}\right)^{\beta}\right) } \\
& \times \sum_{k, m=0}^{\infty} a_{k, m}^{(1+k, n)}, \quad \forall n>-b,
\end{aligned}
$$

where

$$
\Gamma(a, x)=\int_{x}^{\infty} t^{a-1} \exp (-t) d t, \quad x>0,
$$

is the incomplete gamma function, that is

$$
\Gamma(a, x)+\gamma(a, x)=\Gamma(a)
$$

and

$$
\begin{aligned}
\left.\omega_{n}(t)\right|_{\left(\begin{array}{c}
n=1,2, \ldots) \\
(X \leqslant t, t>0)
\end{array}=\right.} & F^{-1}(t) \gamma\left(1+\frac{n}{b},\left(\frac{1}{t}\right)^{\beta}\right) \\
& \times \sum_{k, m=0}^{\infty} a_{k, m}^{(1+k, n)^{(\omega)}}, \quad \forall n>-b,
\end{aligned}
$$

where

$$
a_{k, m}^{(1+k, n)^{(\tau)}}=\omega_{m}^{(1+k, n)} t_{1+k}^{(\tau)}
$$

and 


$$
a_{k, m}^{(1+k, n)^{(\omega)}}=\omega_{m}^{(1+k, n)} t_{1+k}^{(\omega)}
$$

\section{Characterizations}

In this section, we present various characterizations of WG-G distribution. These characterizations are based on: $(i)$ simple relationship between two truncated moments; (ii) the hazard function and (iii) certain functions of the r.v.. One of the advantages of characterization $(i)$ is that the CDF is not required to have a closed form.

We present our characterizations $(i)-($ iii $)$ in the following three subsections.

\subsection{Characterizations based on Ratio of Two Truncated Mo- ments}

In this subsection, we present characterizations of WG-G distribution in terms of a simple relationship between two truncated moments. This characterization result employs a theorem due to Glänzel (1987), see Theorem 1 below. Note that the result holds also when the interval $H$ is not closed. Moreover, as mentioned above, it could be also applied when the CDF $F$ does not have a closed form. As shown in Glänzel (1990), this characterization is stable in the sense of weak convergence.

Theorem 1. Let $(\Omega, \mathcal{F}, \mathbf{P})$ be a given probability space and let $H=[a, b]$ be an interval for some $d<b \quad(a=-\infty, b=\infty$ might as well be allowed $)$. Let $X: \Omega \rightarrow H$ be a continuous r.v. with the distribution function $F$ and let $q_{1}$ and $q_{2}$ be two real functions defined on $H$ such that

$$
\mathbf{E}\left[q_{2}(X) \mid X \geqslant x\right]=\mathbf{E}\left[q_{1}(X) \mid X \geqslant x\right] \eta(x), \quad x \in H,
$$

is defined with some real function $\eta$. Assume that $q_{1}, q_{2} \in C^{1}(H), \eta \in C^{2}(H)$ and $F$ is twice continuously differentiable and strictly monotone function on the set $H$. Finally, assume that the equation $\eta q_{1}=q_{2}$ has no real solution in the interior of $H$. Then $F$ is uniquely determined by the functions $q_{1}, q_{2}$ and $\eta$, particularly

$$
F(x)=\int_{a}^{x} C\left|\frac{\eta^{\prime}(u)}{\eta(u) q_{1}(u)-q_{2}(u)}\right| \exp (-s(u)) d u,
$$


where the function $s$ is a solution of the differential equation $s^{\prime}=\frac{\eta^{\prime} q_{1}}{\eta q_{1}-q_{2}}$ and $C$ is the normalization constant, such that $\int_{H} d F=1$.

Proposition 1. Let $X: \Omega \rightarrow \mathbb{R}$ be a continuous r.v. and let

$$
q_{1}(x)=G(x, \xi)^{\theta(\beta+1)} \exp \left\{\left[\frac{1-\bar{G}(x)^{\theta}}{\bar{G}(x)^{\theta}}\right]^{\beta}\right\}
$$

and

$$
q_{2}(x)=q_{1}(x)\left[1-\bar{G}(x)^{\theta}\right]^{\beta} \text { for } x \in \mathbb{R} .
$$

The r.v. $X$ has PDF (2) if and only if the function $\eta$ defined in Theorem 1 has the form

$$
\eta(x)=\frac{1}{2}\left\{1+\left[1-\bar{G}(x)^{\theta}\right]^{\beta}\right\}, \quad x \in \mathbb{R} .
$$

Proof. Let $X$ be a r.v. with PDF (2), then

$$
(1-F(x)) \mathbf{E}\left[q_{1}(X) \mid X \geqslant x\right]=1-\left[1-\bar{G}(x)^{\theta}\right]^{\beta}, \quad x \in \mathbb{R},
$$

and

$$
(1-F(x)) \mathbf{E}\left[q_{2}(X) \mid X \geqslant x\right]=\frac{1}{2}\left\{1-\left[1-\bar{G}(x)^{\theta}\right]^{2 \beta}\right\}, \quad x \in \mathbb{R},
$$

and finally

$$
\eta(x) q_{1}(x)-q_{2}(x)=\frac{1}{2} q_{1}(x)\left\{1-\left[1-\bar{G}(x)^{\theta}\right]^{\beta}\right\}>0 \text { for } x \in \mathbb{R} .
$$

Conversely, if $\eta$ is given as above, then

$$
s^{\prime}(x)=\frac{\eta^{\prime}(x) q_{1}(x)}{\eta(x) q_{1}(x)-q_{2}(x)}=\frac{\theta \beta g(x) \bar{G}(x)^{\theta-1}\left[1-\bar{G}(x)^{\theta}\right]^{\beta-1}}{1-\left[1-\bar{G}(x)^{\theta}\right]^{\beta}}, \quad x \in \mathbb{R}
$$


and hence

$$
s(x)=-\log \left\{1-\left[1-\bar{G}(x)^{\theta}\right]^{\beta}\right\}, \quad x \in \mathbb{R} .
$$

Now, in view of Theorem $1, X$ has the density (2).

Corollary 1. Let $X: \Omega \rightarrow \mathbb{R}$ be a continuous r.v. and let $q_{1}(x)$ be as in Proposition 1. The PDF of $X$ is (2) if and only if there exist functions $q_{2}$ and $\eta$ defined in Theorem 1 satisfying the differential equation

$$
\frac{\eta^{\prime}(x) q_{1}(x)}{\eta(x) q_{1}(x)-q_{2}(x)}=\frac{\theta \beta g(x) \bar{G}(x)^{\theta-1}\left[1-\bar{G}(x)^{\theta}\right]^{\beta-1}}{1-\left[1-\bar{G}(x)^{\theta}\right]^{\beta}}, \quad x \in \mathbb{R} .
$$

The general solution of the differential equation in Corollary 1 is

$$
\left\{1-\left[1-\bar{G}(x)^{\theta}\right]^{\beta}\right\}^{-1}\left\{-\int\left[\begin{array}{c}
\theta \beta g(x) \bar{G}(x)^{\theta-1}\left[1-\bar{G}(x)^{\theta}\right]^{\beta-1} \\
\times\left(q_{1}(x)\right)^{-1} q_{2}(x)+D
\end{array}\right]\right\}
$$

where $D$ is a constant. Note that a set of functions satisfying the above differential equation is given in Proposition 1 with $D=\frac{1}{2}$. However, it should be also noted that there are other triplets $\left(q_{1}, q_{2}, \eta\right)$ satisfying the conditions of Theorem 1.

Remark 1. Bourguignon et al. (2014), introduced a distribution given by

$$
F(x)=1-\exp \left\{-\alpha\left[\frac{G(x)}{\bar{G}(x)}\right]^{\beta}\right\}, \quad x \in \mathbb{R} .
$$

For $\alpha=1$, the above distribution is a special case of WG-G when $\theta=1$.

\subsection{Characterization based on HRF}

It is known that the hazard function, $h_{F}$, of a twice differentiable distribution function, $F$, satisfies the $1^{\text {st }}$ order differential equation 


$$
\frac{f^{\prime}(x)}{f(x)}=\frac{h_{F}^{\prime}(x)}{h_{F}(x)}-h_{F}(x) .
$$

For many univariate continuous distributions, this is the only characterization available in terms of the hazard function. The following Proposition establishes a non-trivial characterization of WG-G distribution.

Proposition 2. Let $X: \Omega \rightarrow \mathbb{R}$ be a continuous r.v.. The PDF of $X$ is (2) if and only if its hazard function $h_{F}(x)$ satisfies the differential equation

$$
h_{F}^{\prime}(x)-\frac{g^{\prime}(x)}{g(x)} h_{F}(x)=\left\{\frac{\theta \beta\left[(\theta \beta+1)-(\theta+1) \bar{G}(x)^{\theta}\right]}{g(x)^{-2} \bar{G}(x)^{\theta \beta+2}\left[1-\bar{G}(x)^{\theta}\right]^{2-\beta}}\right\}, \quad x \in \mathbb{R} .
$$

Proof. If $X$ has PDF (2), then clearly the above differential equation holds. Now, if the differential equation holds, then

$$
\frac{d}{d x}\left\{g(x)^{-1} h_{F}(x)\right\}=\theta \beta \frac{d}{d x}\left\{\frac{\left[1-\bar{G}(x)^{\theta}\right]^{\beta-1}}{\bar{G}(x)^{\theta \beta+1}}\right\}
$$

or

$$
h_{F}(x)=\frac{\theta \beta g(x)\left[1-\bar{G}(x)^{\theta}\right]^{\beta-1}}{\bar{G}(x)^{\theta \beta+1}},
$$

which is the hazard function of the WG-G distribution.

\subsection{Characterization based on Certain Functions of the r.v.}

The following proposition has already appeared in Hamedani (2013), so we will just state it here which can be used to characterize WG-G distribution. Proposition 3. Let $X: \Omega \rightarrow(d, e)$ be a continuous r.v. with CDF $F$. Let $\psi(x)$ be a differentiable function on $(d, e)$ with $\lim _{x \rightarrow d^{+}} \psi(x)=1$. Then for $\delta \neq 1$,

$$
\mathbf{E}[\psi(X) \mid X \geqslant x]=\delta \psi(x), \quad x \in(d, e),
$$

if and only if 


$$
\psi(x)=(1-F(x))^{\frac{1}{\delta}-1}, \quad x \in(d, e) .
$$

Remark 2. It is easy to see that for certain functions, e.g., $\psi(x)=$ $\exp \left\{-\left[\frac{1-\bar{G}(x)^{\theta}}{\bar{G}(x)^{\theta}}\right]^{\beta}\right\}, \delta=\frac{1}{2}$ and $(d, e)=\mathbb{R}$, Proposition 3 provides a characterization of WG-G distribution. Clearly, there are other suitable functions $\psi$, we chose the above one for simplicity.

\section{Estimation}

\subsection{Maximum Likelihood Estimation}

The maximum likelihood estimators (MLEs) enjoy desirable properties and can be used for constructing confidence intervals and regions and also in test statistics. Let $x_{1}, \ldots, x_{n}$ be an observed random sample from the WG-G distribution with parameters $\beta, \theta$ and $\xi$. Let $\Theta=\left(\beta, \theta, \xi^{\top}\right)^{\top}$ be the $p \times 1$ parameter vector. For determining the MLE of $\Theta$, we have the log-likelihood function

$$
\begin{aligned}
\ell= & \ell(\Theta)=n \log \beta+n \log \theta+\sum_{i=1}^{n} \log g\left(x_{i} ; \xi\right) \sum_{i=1}^{n} \\
& -(\theta \beta+1) \sum_{i=1}^{n} \log \bar{G}\left(x_{i} ; \xi\right)+(\beta-1) \sum_{i=1}^{n} \log d_{i}-\sum_{i=1}^{n} z_{i}^{\beta},
\end{aligned}
$$

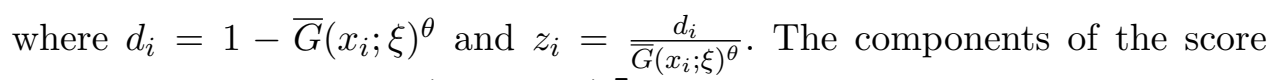
vector, $\mathbf{U}(\Theta)=\frac{\partial \ell}{\partial \Theta}=\left(\frac{\partial \ell}{\partial \beta}, \frac{\partial \ell}{\partial \theta}, \frac{\partial \ell}{\partial \xi}\right)^{\top}$ are given in Appendix A. Setting the nonlinear system of equations $U_{\beta}=U_{\theta}=0$ and $U_{\xi}=\mathbf{0}$ and solving them simultaneously yields the $\operatorname{MLE} \widehat{\Theta}=\left(\widehat{\beta}, \widehat{\theta}, \widehat{\xi^{\top}}\right)^{\top}$. The interval estimation of the model parameters (for the WGW model as a special case) requires the $3 \times 3$ observed information matrix $J(\theta)=\left\{J_{i j}\right\}$ for $i, j=\beta, \theta, b$. The multivariate normal $N_{3}\left(0, J(\widehat{\theta})^{-1}\right)$ distribution, under standard regularity conditions, can be used to provide approximate confidence intervals for the unknown parameters, where $J(\widehat{\theta})$ is the total observed information matrix evaluated at $\widehat{\theta}$. Then, approximate $100(1-\delta) \%$ confidence intervals for $\beta, \theta$ and $b$ can be determined by: $\widehat{\beta} \pm z_{\delta / 2} \sqrt{\widehat{J}_{\beta \beta}}, \quad \widehat{\theta} \pm z_{\delta / 2} \sqrt{\widehat{J}_{\theta \theta}}$ and $\widehat{b} \pm z_{\delta / 2} \sqrt{\widehat{J}_{b b}}$ where $z_{\delta / 2}$ is the upper $(\delta / 2)$ th percentile of the standard normal model. 
The elements of the observed information matrix are given in Appendix B. Overwhelmingly with lifetime data, we encounter censored observations. There are different forms of censoring: type I censoring, type II censoring, etc. Here, we will consider the general case of the multi-censored data: there are $n$ subjects of which $n_{0}$ are known to have failed at the times $x_{1}, \ldots, x_{n_{0}}, n_{1}$ are known to have failed in the interval $\left[s_{j-1}, s_{j}\right], j=1, \ldots, n_{1}, n_{2}$ survived to a time $r_{j}, j=1, \ldots, n_{2}$ but not observed any longer, where $n_{0}+n_{1}+n_{2}=n$ and that type $\mathbf{I}$ censoring and type II censoring are contained as particular cases of multi-censoring. The log-likelihood function for $\boldsymbol{\Theta}$ is

$$
\begin{aligned}
\ell_{n}(\boldsymbol{\Theta})= & n \log (\beta)+n \log (\theta)+\sum_{i=1}^{n_{0}} \log g\left(x_{i} ; \xi\right) \\
& -(\theta \beta+1) \sum_{i=1}^{n_{0}} \log \bar{G}\left(x_{i} ; \xi\right)-\sum_{i=1}^{n_{2}}\left[\frac{1-\bar{G}\left(r_{i}, \xi\right)^{\theta}}{\bar{G}\left(r_{i}, \xi\right)^{\theta}}\right]^{\beta} \\
& +\sum_{i=1}^{n_{1}} \log \left[-\left(1-\exp \left\{-\left[\frac{1-\bar{G}\left(s_{i}, \xi\right)^{\theta}}{\bar{G}\left(s_{i}, \xi\right)^{\theta}}\right]^{\beta}\right\}\right)\right] \\
& \left.\left.+(\beta-1) \sum_{i=1}^{n_{0}} \log \left[1-\bar{G}\left(x_{i} ; \xi\right)^{\theta}\right]-\sum_{i=1}^{n_{2}}\left[\frac{1-\bar{G}\left(s_{i-1}, \xi\right)^{\theta}}{\bar{G}\left(s_{i-1}, \xi\right)^{\theta}}\right]\right)\right]
\end{aligned}
$$

The normal equations are given in Appendix C.

\subsection{Simulation Studies}

We used computer software R Core Team (2016) for the simulation study. For each combination of specific parameters, we simulated a sample data. MLEs are computed based on this data using R function optimx (see John and Ravi, 2011). To maximize the log likelihood function, Nelder and Mead (1965) method is used as it provides more robust results than other methods. Finally, standard errors (SE) of the estimates are obtained from the Hessian matrix provided by optimx. All values in this study are generated by using $R$ package ggplot2 (see Hadley, 2009). 


\subsubsection{Choice of Parameters}

A thorough investigation has been carried through to pick the parameters for the simulation study. We observed that for WGW density $\beta=0.5,1,1.5$, $\theta=0.5,1,1.5$ and $b=1,2$ and 3 produce most different varieties of shapes as shown in Figure 3. Thus we chose to run the simulation based on the 27 combinations of these parameters. For each simulation sample sizes $n=200$, 300 are considered. The densities in Figure 3 are used to simulate data based on which parameters are estimated with their standard deviations as shown in Table 4. For Weibull Generalized Lindley (WGLi) distribution we considered $\beta=0.5,1,1.5$ and $\alpha=1,2,3$. For each simulation sample sizes $n=200,300$ are considered as well. The densities of all these 9 parameters are shown in Figure 4. The densities in Figure 3 are used to simulate data based on which parameters are estimated with their standard deviations as shown in Table 5.

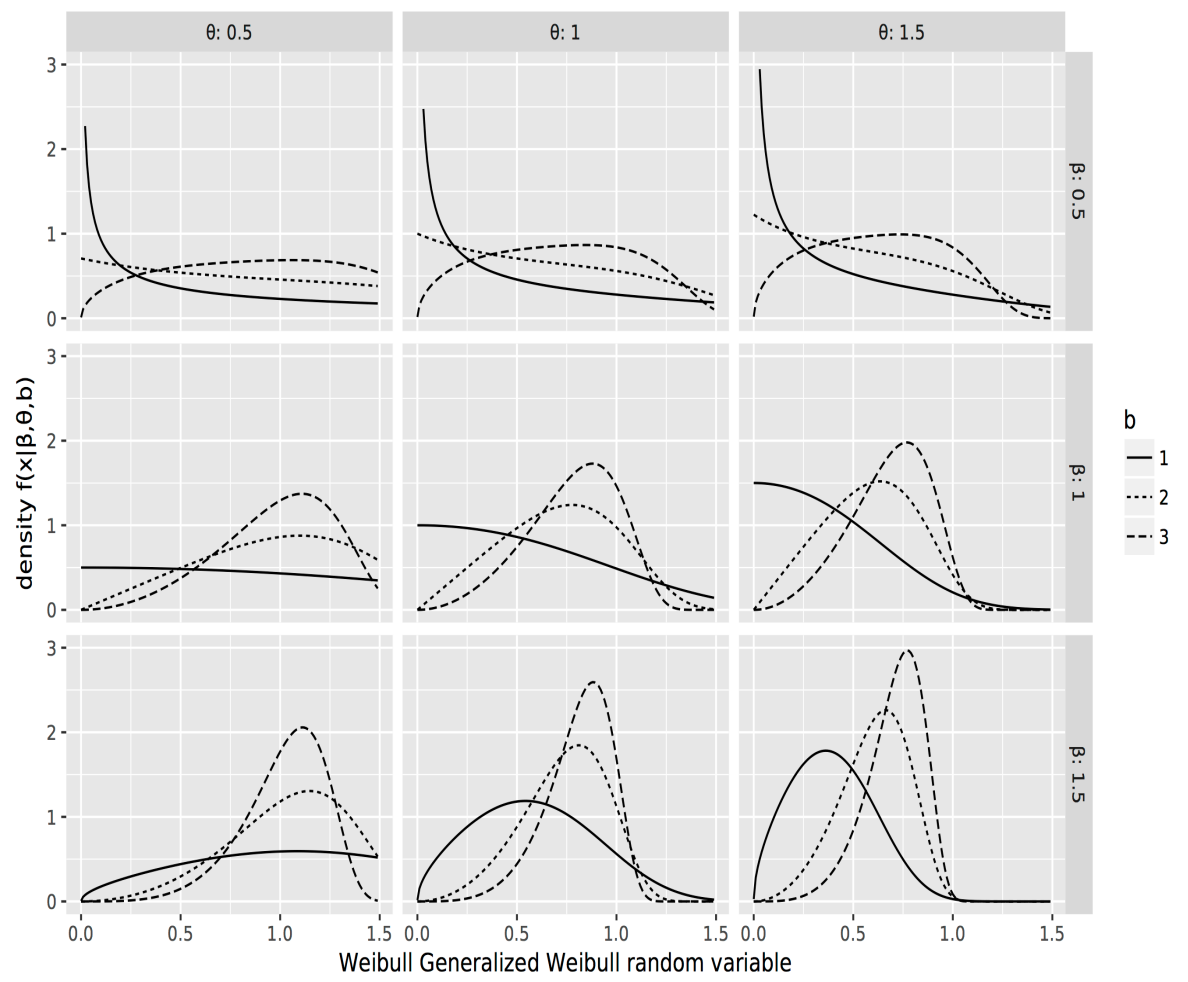

Figure 3. WGW densities for different combinations of parameter $\beta, \theta$ and $b$. 


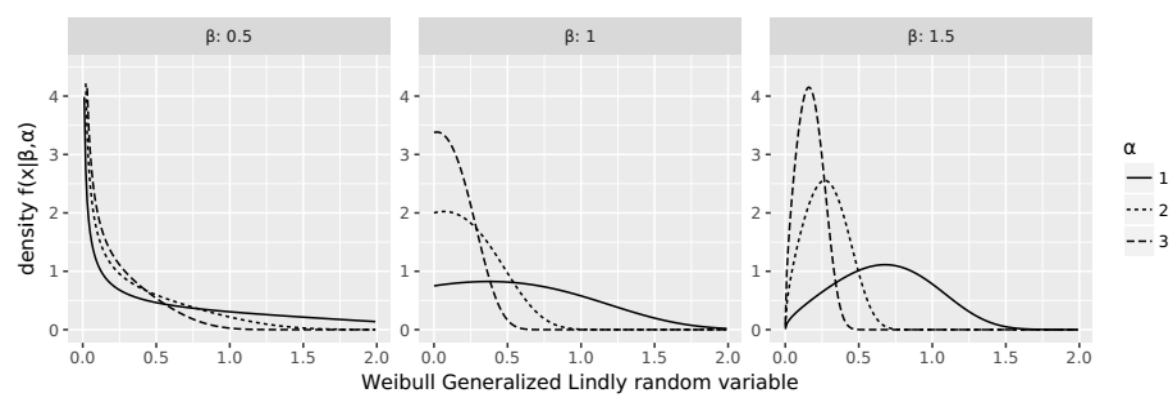

Figure 4. WGLi densities for different combinations of parameter $\beta$ and $\alpha$.

\subsubsection{Simulation Results}

The results of the simulated study for WGW distribution are shown in Figure 4 and presented in Tables 4 and 5. For WGLi distribution, the results are shown in Table 6. Tables 4-6 are given in Appendix D. Actual true PDF (continuous line) is overlaid along with PDF generated using MLE (dotted line). The parameters and their standard errors (SE) are shown in Tables 4 and 5 .

\section{Data Analysis}

In this section, we provide three applications to real data to illustrate the importance of the WGW family presented in Section 1. The MLEs of the parameters for these models are calculated and three goodness-of-fit statistics are used to compare the new family with its sub-models. We compared the fits of the WGW distribution with some of its special cases and other models such as Weibull (W) (Weibull, 1951), exponentiated Weibull (EW) (Mudholkar and Srivastava, 1993 and Mudholkar et al., 1995), Kumaraswamy Weibull (KwW) (Cordeiro et al., 2010), beta Weibull (BW) (Lee et al., 2007),Transmuted Weibull (TW) (Aryal and Tsokos, 2011) and McDonald Weibull (McW) (Cordeiro et al., 2014) distributions given by:

- W:

$$
f(x)=b x^{b-1} \exp \left(-x^{b}\right)
$$




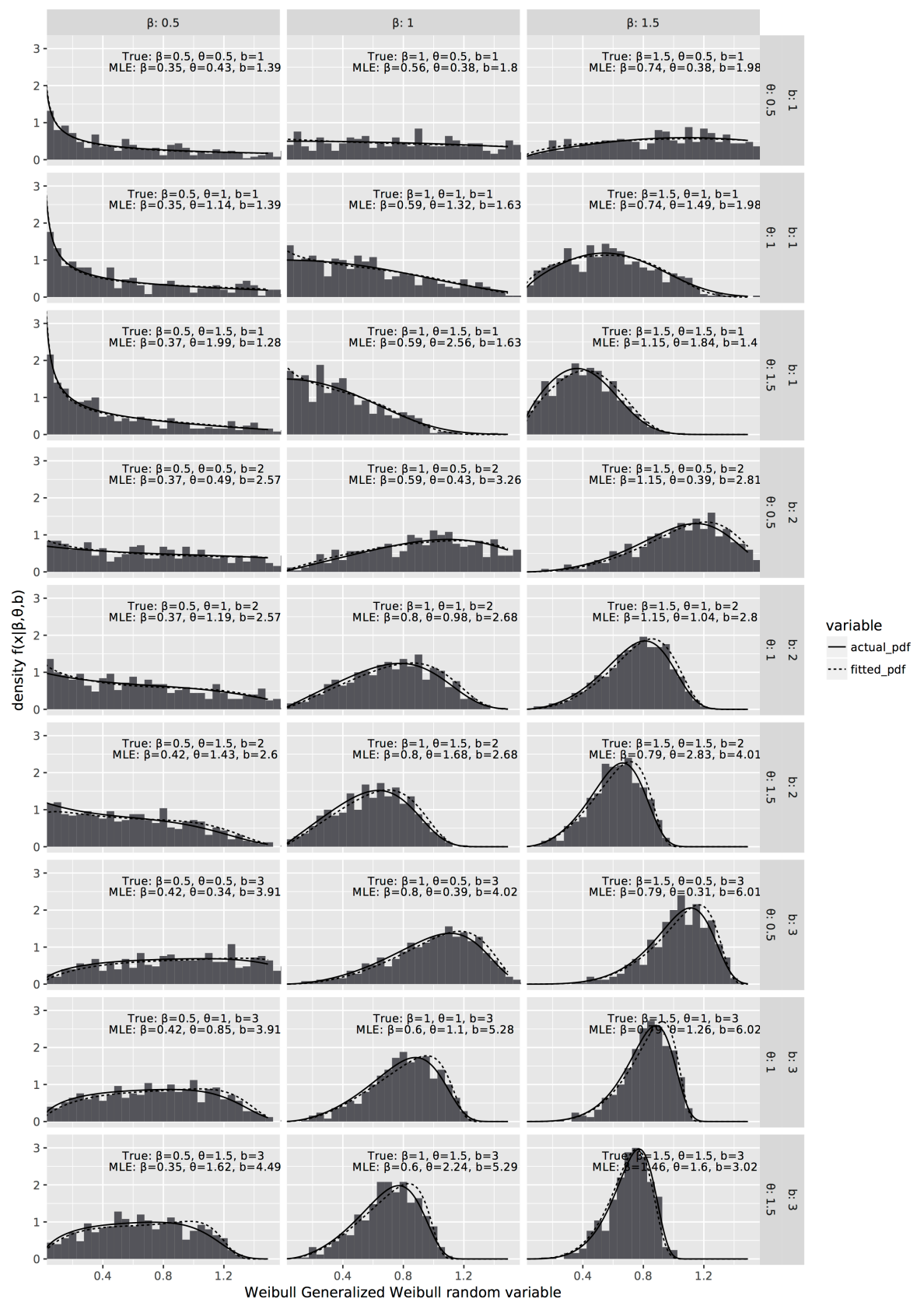

Figure 5. Histograms of simulated data from WGW densities for different combinations of parameters. 
- EW:

$$
f(x)=\alpha b x^{b-1}\left[1-\exp \left(-x^{b}\right)\right]^{\alpha-1} \exp \left(-x^{b}\right),
$$

- BW:

$$
f(x)=b x^{b-1} \exp \left(-\beta x^{b}\right)\left[1-\exp \left(-x^{b}\right)\right]^{\alpha-1} \mathrm{~B}^{-1}(\alpha, \beta),
$$

- $\mathrm{KwW}$ :

$$
\begin{aligned}
f(x)= & \alpha \beta b x^{b-1} \exp \left(-x^{b}\right)\left[1-\exp \left(-x^{b}\right)\right]^{\alpha-1} \\
& \times\left\{1-\left[1-\exp \left(-x^{b}\right)\right]^{\alpha}\right\}^{\beta-1}
\end{aligned}
$$

- TW:

$$
f(x)=b x^{b-1} \exp \left(-\alpha x^{b}\right)\left\{1+\lambda-2 \lambda\left[1-\exp \left(-\alpha x^{b}\right)\right]\right\},
$$

- $\mathrm{McW}$ :

$$
\begin{aligned}
f(x)= & \gamma b x^{b-1} \exp \left(-\beta x^{b}\right)\left[1-\exp \left(-x^{b}\right)\right]^{\alpha \gamma-1} \\
& \times\left\{1-\left[1-\exp \left(-x^{b}\right)\right]^{\gamma}\right\}^{\beta-1} \mathrm{~B}^{-1}(\alpha, \beta) .
\end{aligned}
$$

The $1^{\text {st }}$ data set consists of failure times for a particular windshield model including 88 observations that are classified as failed times of windshields. These data were previously studied by Murthy et al. (2004). The $2^{\text {nd }}$ real data set represents the survival times of 121 patients with breast cancer obtained from a large hospital in a period from 1929 to 1938 (Lee, 1992). The data was examined by Ramos et al. (2013). The $3^{\text {rd }}$ real data set corresponds to an uncensored data set from Nichols and Padgett (2006) on breaking stress of carbon fibres (in Gba). In order to compare the fitted models, we consider some goodness-of-fit measures including the Akaike information criterion $(A I C)$ and Bayesian information criterion $(B I C)$ as 
Table 1. The MLEs and the goodness-of-fit statistics for the $1^{\text {st }}$ data set.

\begin{tabular}{llrrrc}
\hline \multicolumn{1}{c}{ Distribution } & Parameter Estimates & AIC & BIC & K-S & p-value \\
\hline $\mathrm{W}(b)$ & 2.562 & 331.9 & 334.4 & 0.303 & 0.000 \\
$\mathrm{EW}(b, \alpha)$ & $1.316,3.595$ & 286.7 & 291.6 & 0.121 & 0.163 \\
$\mathrm{BW}(b, \alpha, \beta)$ & $1464.1,3.52,2014.8$ & 282.7 & 290.1 & 0.103 & 0.319 \\
$\mathrm{KwW}(b, \alpha, \beta)$ & $80.66,2.41,3351.1$ & 268.9 & 276.2 & 0.069 & 0.816 \\
$\mathrm{TW}(b, \lambda)$ & $1.749,-0.996$ & 297.6 & 302.5 & 0.185 & 0.050 \\
$\mathrm{McW}(b, \alpha, \beta, \gamma)$ & $3.73,0.256,27.80,8.68$ & 269.2 & 279.0 & 0.076 & 0.703 \\
$\mathrm{WGW}(b, \theta, \beta)$ & $7.12,1.68,1.79$ & 264.1 & 271.4 & 0.066 & 0.847 \\
\hline
\end{tabular}

$$
A I C=-2 \hat{\ell}+2 p \text { and } B I C=-2 \hat{\ell}+p \log (n),
$$

where $p$ is the number of parameters, $n$ is the sample size and $\hat{\ell}$ is the log-likelihood function evaluated at the MLEs. The smaller the values of these statistics, the better are the fits. Upper tail percentiles of the asymptotic distributions of these goodness-of-fit statistics were tabulated in Nichols and Padgett (2006). Tables 1, 2 and 3 list the MLEs of the model parameters and the numerical values of the model selection statistics $A I C, B I C, A^{*}, W^{*}$ and $\mathrm{K}-\mathrm{S}$. We note from the values in Table 3 that the WGW model has the lowest values of the $A I C$, $B I C, A^{*}, W^{*}$ and K-S statistics (for the $1^{\text {st }}$ data set) as compared to their submodels, suggesting that the WGW model provide the best fit. The histogram of the $1^{\text {st }}$ data and the estimated PDFs and estimated CDFs (ECDFs) of the WGW model and its sub-models are displayed in Figure 6. Similarly, it is also evident from Table 2 that the WGW gives the lowest values the $A I C, B I C$ and $\mathrm{K}-\mathrm{S}$ statistics (for the $2^{\text {nd }}$ data set) as compared to their sub-models, and therefore these models can be chosen as the best ones. The histogram of the $2^{\text {nd }}$ data and estimated PDFs and CDFs of the WGW distribution and its sub-models are displayed in Figure 7 . In addition, it is clear from Table 3 that the WGW gives the lowest values the $A I C, B I C$ and $\mathrm{K}-\mathrm{S}$ statistics (for the $3^{\text {rd }}$ data set) as compared to their sub-models, and therefore these models can be chosen as the best ones. The histogram of the $3^{\text {rd }}$ data and estimated PDFs and CDFs of the WGW distribution and its sub-models are displayed in Figure 8. 

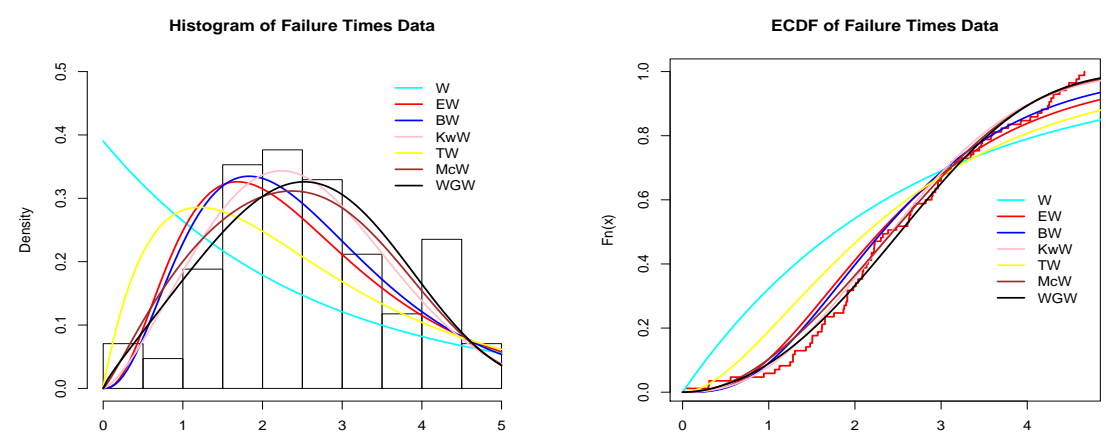

Figure 6. Histogram (left) and ECDF (right) of the failure times data.

Table 2. The MLEs and the goodness-of-fit statistics for the $2^{\text {nd }}$ data set.

\begin{tabular}{llcccc}
\hline \hline \multicolumn{1}{c}{ Distribution } & Parameter Estimates & AIC & BIC & K-S & p-value \\
\hline $\mathrm{W}(b)$ & 46.35 & 1172.2 & 1175.1 & 0.120 & 0.061 \\
$\mathrm{EW}(b, \alpha)$ & $36.03,1.515$ & 1166.1 & 1173.7 & 0.080 & 0.407 \\
$\mathrm{BW}(b, \alpha, \beta)$ & $12635.4,1.492,406.1$ & 1165.6 & 1173.9 & 0.076 & 0.490 \\
$\mathrm{McW}(b, \alpha, \beta, \gamma)$ & $169.4,0.508,9.05,2.28$ & 1166.0 & 1177.2 & 0.058 & 0.847 \\
$\mathrm{WGW}(b, \theta, \beta)$ & $10.06,0.126,0.957$ & 1165.5 & 1172.8 & 0.055 & 0.865 \\
\hline
\end{tabular}
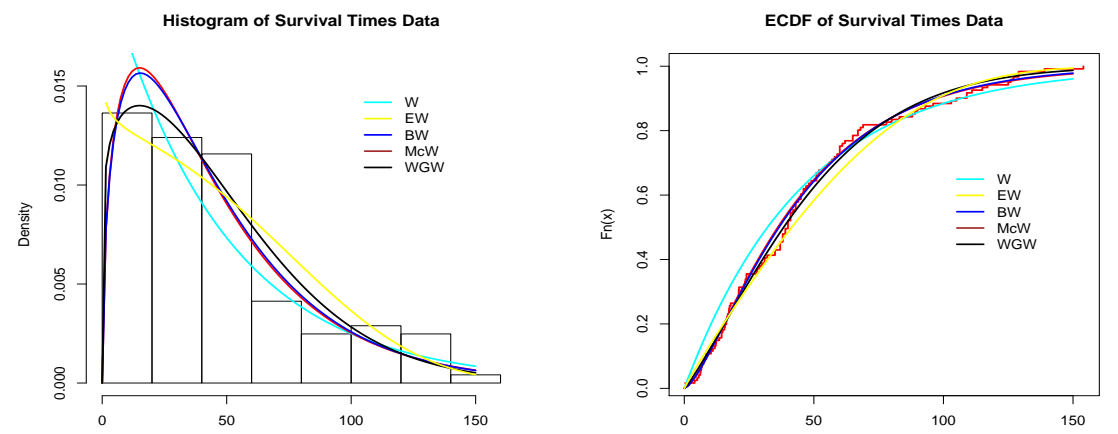

Figure 7. Histogram (left) and ECDF (right) of the survival times data. 
Table 3. The MLEs and the goodness-of-fit statistics for the $3^{\text {st }}$ data set.

\begin{tabular}{llcccc}
\hline \multicolumn{1}{c}{ Distribution } & \multicolumn{1}{c}{ Parameter Estimates } & AIC & BIC & K-S & p-value \\
\hline $\mathrm{W}(b)$ & 2.261 & 394.7 & 397.3 & 0.321 & 0.000 \\
$\mathrm{EW}(b, \alpha)$ & $0.987,7.788$ & 296.3 & 301.5 & 0.107 & 0.196 \\
$\mathrm{BW}(b, \alpha, \beta)$ & $1008.8,5.953,2288.6$ & 292.4 & 300.2 & 0.093 & 0.346 \\
$\mathrm{KwW}(b, \alpha, \beta)$ & $47.941,2.883,3420.7$ & 288.9 & 296.7 & 0.059 & 0.871 \\
$\mathrm{TW}(b, \lambda)$ & $1.307,-0.92$ & 304.1 & 309.4 & 0.184 & 0.003 \\
$\mathrm{McW}(b, \alpha, \beta, \gamma)$ & $10.791,1.033,92.96,3.124$ & 290.6 & 301.0 & 0.064 & 0.806 \\
$\mathrm{WGW}(b, \theta, \beta)$ & $5.197,1.196,2.017$ & 287.4 & 300.2 & 0.055 & 0.892 \\
\hline
\end{tabular}
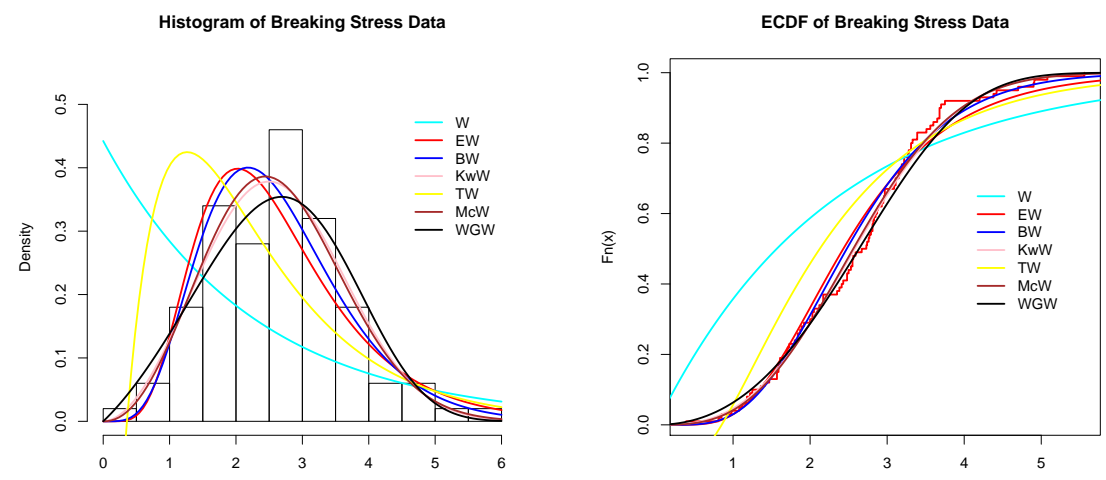

Figure 8. Histogram (left) and ECDF (right) of the breaking stress data.

\section{Conclusions}

A new class of distributions called the Weibull Generalized-G family with two extra positive shape parameters is introduced and studied. We provide some mathematical properties of the new family including ordinary and incomplete moments, generating function, stress-strength model, residual life and reversed residual life functions. Characterizations based on a ratio of two truncated moments, in terms of hazard function and based on certain functions of the r.v. are presented. The maximum likelihood method is used for estimating the model parameters. We assessed the performance of the maximum likelihood estimators in terms of biases and mean squared errors by means of a simulation study. Finally, the usefulness of the family is illus- 
trated by means of three different real data sets. The new models provide consistently better fits than other competitive models for these data sets.

\section{Acknowledgment}

The authors gratefully acknowledge with thanks the very thoughtful and constructive comments and suggestions of the Editor-in-Chief and the four reviewers which resulted in much improved paper.

\section{References}

Afify, A.Z., Alizadeh, M., Yousof, H.M., Aryal, G. and Ahmad, M. (2016a). The Transmuted Geometric-G Family of Distributions: Theory and Applications. Pak. J. Statist., 32, 139-160.

Afify, A.Z., Cordeiro, G.M., Yousof, H.M., Alzaatreh, A. and Nofal, Z.M. (2016b). The Kumaraswamy Transmuted-G Family of Distributions: Properties and Applications. J. Data Sci., 14, 245-270.

Afify, A.Z., Yousof, H.M. and Nadarajah, S. (2017). The Beta Transmuted-H Family of Distributions: Properties and Applications. Statistics and Its Inference, 10, 505-520.

Alizadeh, M., Ghosh, I., Yousof, H.M., Rasekhi, M. and Hamedani G.G. (2017a). The Generalized Odd Generalized Exponential Family of Distributions: Properties, Characterizations and Applications, J. Data Sci., 15, 443-466.

Alizadeh, M., Lak, F., Rasekhi, M., Ramires, T.G., Yousof, H.M. and Altun, E. (2018). The Odd Log-logistic Topp Leone G Family of Distributions: Heteroscedastic Regression Models and Applications. Computational Statistics, forthcoming.

Alizadeh, M., Rasekhi, M., Yousof, H.M. and Hamedani G.G. (2017b). The Transmuted Weibull G Family of Distributions. Hacettepe Journal of Mathematics and Statistics, forthcoming.

Alizadeh, M., Yousof, H.M., Afify A.Z., Cordeiro, G.M. and Mansoor, M. (2016). The Complementary Generalized Transmuted Poisson-G Family. Austrian Journal of Statistics, 47, 51-71.

Alzaatreh, A., Famoye, F., Lee, C. (2013). A New Method for Generating Families of Continuous Distributions. Metron, 71, 63-79.

Aryal, G.R. and Tsokos, C.P. (2011). Transmuted Weibull Distribution: a Generalization of the Weibull Probability Distribution. European Journal of Pure and Applied Mathematics, 4, 89-102. 
Barreto-Souza, W. and Simas, A.B. (2013). The Exp-G Family of Probability Distributions. Braz. J. Prob. Stat., 27, 84-109.

Bourguignon, M., Silva, R.B. and Cordeiro, G.M. (2014). The Weibull-G Family of Probability Distributions, J. Data Sci., 12, 53-68.

Brito, E., Cordeiro, G.M., Yousof, H.M., Alizadeh, M. and Silva, G.O. (2017). Topp-Leone Odd Log-Logistic Family of Distributions, Journal of Statistical Computation and Simulation, 87, 3040-3058.

Cordeiro, G.M., Afify, A.Z., Yousof, H.M., Pescim, R.R. and Aryal, G.R. (2017). The Exponentiated Weibull-H Family of Distributions: Theory and Applications. Mediterranean Journal of Mathematics, 14, 1-22.

Cordeiro, G.M., Hashimoto, E.M., Edwin, E.M.M. Ortega, E.M. (2014). The McDonald Weibull Model. Statistics: A Journal of Theoretical and Applied Statistics, 48, 256-278.

Cordeiro, G.M., Ortega, E.M. and Nadarajah, S. (2010). The Kumaraswamy Weibull Distribution with Application to Failure Data. Journal of the Franklin Institute, 347, 1399-1429.

Cordeiro, G.M., Yousof, H.M., Ramires, T.G. and Ortega, E.M. (2018). The Burr XII System of Densities: Properties, Regression Model and Applications. Journal of Statistical Computation and Simulation, 88, 432-456.

Glänzel, W. (1987). A Characterization Theorem based on Truncated Moments and Its Application to Some Distribution Families, Mathematical Statistics and Probability Theory (Bad Tatzmannsdorf, 1986), Vol. B, Reidel, Dordrecht, 75-84.

Glänzel, W. (1990). Some Consequences of a Characterization Theorem based on Truncated Moments, Statistics: A Journal of Theoretical and Applied Statistics, 21, 613-618.

Gupta, R.C., Gupta, P.L. and Gupta, R.D. (1998). Modeling Failure Time Data by Lehmann Alternatives. Commun. in Stat. Theory Methods, 27, 887-904.

Hamedani, G.G. (2013). On Certain Generalized Gamma Convolution Distributions II, Technical Report, No. 484, MSCS, Marquette University.

Hamedani, G.G. Yousof, H.M., Rasekhi, M., Alizadeh, M., Najibi, S.M. (2017). Type I general exponential class of distributions. Pak. J. Stat. Oper. Res., XIV(1), 39-55.

Hamedani, G.G. Rasekhi, M., Najibi, S.M., Yousof, H.M. and Alizadeh, M. (2018). Type II General Exponential Class of Distributions. Pak. J. Stat. Oper. Res., forthcoming.

John, C.N. and Ravi, V. (2011). Unifying Optimization Algorithms to Aid Software System Users: Optimx for R. Journal of Statistical Software, 43, 1-14. URL http://www.jstatsoft.org/v43/i09/. 
Korkmaz, M.C. and Genc, A.I. (2017). A New Generalized Two-sided Class of Distributions with an Emphasis on Two-sided Generalized Normal Distribution. Commun. in Stat. Simu. and Compu. DOI: 10.1080/03610918.2015.1005233, forthcoming.

Korkmaz, M.C., Cordeiro, G.M., Yousof, H.M. Pescim, R.R., Afify, A.Z. and Nadarajah, S. (2018a). The Weibull Marshall-Olkin Family: Regression Model and Applications to Censored Data, Commun. in Stat. Theory Methods, forthcoming.

Korkmaz, M.C., Yousof, H.M., Rasekhi, M. and Hamedani, G.G. (2017). The Exponential Lindley Odd Log-logistic G Family: Properties, Characterizations and Applications. Journal of Statistical Theory and Applications, forthcoming.

Korkmaz, M.C., Yousof, H.M., Hamedani, G.G. and Ali, M.M. (2018b). The Marshall-Olkin Generalized G Poisson Family of Distributions, Pakistan Journal of Statistics, 34, 251-267.

Lee, C., Famoye, F. and Olumolade, O. (2007). Beta-Weibull Distribution: Some Properties and Applications to Censored Data. Journal of Modern Applied Statistical Methods, 6, 17.

Lee, E.T. (1992). Statistical Methods for Survival Data Analysis. John Wiley, New York.

Marshall, A.W. and Olkin, I. (1997). A New Method for Adding a Parameter to a Family of Distributions with Application to the Exponential and Weibull Families. Biometrika, 84, 641-652.

Merovci, F., Alizadeh, M., Yousof, H.M. and Hamedani, G.G. (2017). The Exponentiated Transmuted-G Family of Distributions: Theory and Applications, Commun. in Stat. Theory Methods, 46, 10800-10822.

Mudholkar, G.S. and Srivastava, D.K. (1993). Exponentiated Weibull Family for Analyzing Bathtub Failure Rate Data. IEEE Transactions on Reliability, 42, 299-302.

Mudholkar, G.S., Srivastava, D.K. and Freimer, M. (1995). The Exponentiated Weibull Family: a Reanalysis of the Bus-motor-failure Data. Technometrics, 37, 436-445.

Murthy, D.N.P., Xie, M., Jiang, R. (2004). Weibull Models, volume 505. John Wiley \& Sons.

Nelder, J.A. and Mead, R. (1965). A Simplex Method for Function Minimization. The Computer Journal, 7, 308-313.

Nofal, Z.M., Afify, A.Z., Yousof, H.M. and Cordeiro, G.M. (2017). The Generalized Transmuted-G Family of Distributions. Commun. in Stat. Theory Methods, 46, 4119-4136.

Nichols, M.D. and Padgett, W.J. (2006). A Bootstrap Control Chart for Weibull Percentiles. Quality and Reliability Engineering International, 22, 141-151.

Ramos, M.W.A, Cordeiro, G.M., Marinho, P.R.D., Dias, C.R.B. and Hamedani, G.G. (2013). The Zografos-Balakrishnan Log-logistic Distribution: Properties and Applications. J. Stat. Theory Appl., 12, 225-244. 
R Core Team (2016). R: A Language and Environment for Statistical Computing. R Foundation for Statistical Computing, Vienna, Austria. URL https://www.R-project.org/.

Tahir, M.H., Zubair, M., Mansoor, M., Cordeiro, G.M., Alizadeh, M. and Hamedani, G.G. (2016). A New Weibull-G Family of Distributions. Hacet. J. Math. Stat., forthcoming.

Weibull, W. (1951). A Statistical Distribution Function of Wide Applicability. J. Appl. Mech. Trans, 18, 293-297.

Yousof, H.M., Afify, A.Z., Alizadeh, M., Butt, N.S., Hamedani, G.G. and Ali, M.M. (2015). The Transmuted Exponentiated Generalized-G Family of Distributions, Pak. J. Stat. Oper. Res., 11, 441-464.

Yousof, H.M., Afify, A.Z., Hamedani, G.G. and Aryal, G. (2016). The Burr X Generator of Distributions for Lifetime Data. Journal of Statistical Theory and Applications, 16, 288-305.

Yousof, H.M., Altun, E., Ramires, T.G., Alizadeh, M. and Rasekhi, M. (2018a). A New Family of Distributions with Properties, Regression Models and Applications, Journal of Statistics and Management Systems, 21, 163-188.

Yousof, H.M., Alizadeh, M., Jahanshahiand, S.M.A., Ramires, T. G., Ghosh, I. and Hamedani, G.G. (2017a). The Transmuted Topp-Leone G Family of Distributions: Theory, Characterizations and Applications, Journal of Data Science. 15, 723-740.

Yousof, H.M., Rasekhi, M., Afify, A.Z., Alizadeh, M., Ghosh, I. and Hamedani, G.G. (2017b). The Beta Weibull-G Family of Distributions: Theory, Characterizations and Applications, Pakistan Journal of Statistics, 33, 95-116.

Yousof, H.M., Rasekhi, M., Altun, E. and Alizadeh, M. (2018b). The Extended Odd Frechet Family of Distributions: Properties, Applications and Regression Modeling, Journal of Data Science, forthcoming.

\section{Appendix A}

The components of the score vector

$$
\begin{gathered}
U_{\beta}=\frac{n}{\beta}-\theta \sum_{i=1}^{n} \log \bar{G}\left(x_{i} ; \xi\right)+\sum_{i=1}^{n} \log d_{i}-\sum_{i=1}^{n} \frac{\log z_{i}}{z_{i}^{-\beta}}, \\
U_{\theta}=\frac{n}{\theta}-\beta \sum_{i=1}^{n} \log \bar{G}\left(x_{i} ; \xi\right)+(\beta-1) \sum_{i=1}^{n} \frac{m_{i}}{d_{i}}-\beta \sum_{i=1}^{n} \frac{p_{i}}{z_{i}^{1-\beta}},
\end{gathered}
$$

and (for $r=1, \ldots, q$ ) 


$$
\begin{aligned}
U_{\xi_{r}}= & \sum_{i=1}^{n} \frac{g_{r}^{\prime}\left(x_{i} ; \xi\right)}{g\left(x_{i} ; \xi\right)}+(\theta b+1) \sum_{i=1}^{n} \frac{G_{r}^{\prime}\left(x_{i} ; \xi\right)}{\bar{G}\left(x_{i} ; \xi\right)} \\
& +(\beta-1) \sum_{i=1}^{n} \frac{w_{i, r}}{d_{i}}-\beta \sum_{i=1}^{n} \frac{q_{i, r}}{z_{i}^{1-\beta}}
\end{aligned}
$$

where

$$
\begin{aligned}
m_{i} & =-\bar{G}\left(x_{i} ; \xi\right)^{\theta} \log \bar{G}\left(x_{i} ; \xi\right), g_{r}^{\prime}\left(x_{i} ; \xi\right)=\partial g\left(x_{i} ; \xi\right) / \partial \xi_{r} \\
w_{i, r} & =\theta \bar{G}\left(x_{i} ; \xi\right)^{\theta-1} G_{r}^{\prime}\left(x_{i} ; \xi\right), G_{r}^{\prime}\left(x_{i} ; \xi\right)=\partial G\left(x_{i} ; \xi\right) / \partial \xi_{r} \\
q_{i, r} & =\bar{G}\left(x_{i} ; \xi\right)^{-\theta}\left[w_{i, r}-\theta s_{i} G_{r}^{\prime}\left(x_{i} ; \xi\right) \bar{G}\left(x_{i} ; \xi\right)^{-1}\right], \text { and } \\
p_{i} & =\bar{G}\left(x_{i} ; \xi\right)^{-\theta}\left[m_{i}-d_{i} \log \bar{G}\left(x_{i} ; \xi\right)\right] .
\end{aligned}
$$

\section{Appendix B}

The elements of the observed information matrix are

$$
\begin{gathered}
U_{\beta \beta}=\frac{-n}{\beta^{2}}-\sum_{i=1}^{n} \frac{\left(\log z_{i}\right)^{2}}{z_{i}^{-\beta}}, \\
U_{\beta \theta}=-\sum_{i=1}^{n} \log \bar{G}\left(x_{i} ; \xi\right)+\sum_{i=1}^{n} \frac{m_{i}}{d_{i}}-\sum_{i=1}^{n} \frac{1+\beta \log z_{i}}{p_{i}^{-1} z_{i}^{-\beta+1}}, \\
U_{\beta \xi_{r}}=\theta \sum_{i=1}^{n} \frac{G_{r}^{\prime}\left(x_{i} ; \xi\right)}{\bar{G}\left(x_{i} ; \xi\right)}+\sum_{i=1}^{n} \frac{w_{i, r}}{d_{i}}-\sum_{i=1}^{n} \frac{1+\beta \log z_{i}}{q_{i, r} z_{i}^{-\beta+1}}, \\
U_{\theta \theta}=\frac{-n}{\theta^{2}}+(\beta-1) \sum_{i=1}^{n} \frac{-d_{i} \bar{G}\left(x_{i} ; \xi\right)^{\theta}\left[\log \bar{G}\left(x_{i} ; \xi\right)\right]^{2}-m_{i}^{2}}{d_{i}} \\
-\beta \sum_{i=1}^{n}\left[\frac{(\beta-1)}{p_{i}^{-2} z_{i}^{2-\beta}}+\left(\frac{\partial p_{i}}{\partial \theta}\right) z_{i}^{\beta-1}\right],
\end{gathered}
$$




$$
\begin{aligned}
U_{\theta \xi_{r}}= & \beta \sum_{i=1}^{n} \frac{G_{r}^{\prime}\left(x_{i} ; \xi\right)}{\bar{G}\left(x_{i} ; \xi\right)}-\beta \sum_{i=1}^{n}\left[\frac{(\beta-1)}{z_{i}^{2-\beta}\left(p_{i} q_{i, r}\right)^{-1}}+\frac{\left(\frac{\partial p_{i}}{\partial \xi_{r}}\right)}{z_{i}^{1-\beta}}\right] \\
& +(\beta-1) \sum_{i=1}^{n}\left\{\frac{G_{r}^{\prime}\left(x_{i} ; \xi\right) \bar{G}\left(x_{i} ; \xi\right)^{\theta-1}}{d_{i}\left[1+\theta \log \bar{G}\left(x_{i} ; \xi\right)\right]^{-1}}-\frac{m_{i} w_{i, r}}{d_{i}}\right\},
\end{aligned}
$$

and

$$
\begin{aligned}
U_{\xi_{r} \xi_{r}}= & \sum_{i=1}^{n} \frac{g\left(x_{i} ; \xi\right) g_{r}^{\prime \prime}\left(x_{i} ; \xi\right)-\left[g_{r}^{\prime}\left(x_{i} ; \xi\right)\right]^{2}}{g\left(x_{i} ; \xi\right)^{2}} \\
& +(\theta b+1) \sum_{i=1}^{n} \frac{\bar{G}\left(x_{i} ; \xi\right) G_{r}^{\prime \prime}\left(x_{i} ; \xi\right)+\left[G_{r}^{\prime}\left(x_{i} ; \xi\right)\right]^{2}}{\bar{G}\left(x_{i} ; \xi\right)^{2}} \\
& +(\beta-1) \sum_{i=1}^{n} \frac{d_{i}\left(\partial w_{i, r} / \partial \xi_{r}\right)-w_{i, r}}{d_{i}} \\
& -\beta \sum_{i=1}^{n}\left[\frac{(\beta-1) q_{i, r}}{z_{i}^{2-\beta}}+\frac{\left(\partial q_{i, r} / \partial \xi_{r}\right)}{z_{i}^{1-\beta}}\right]
\end{aligned}
$$

where

$$
g_{r}^{\prime \prime}\left(x_{i} ; \xi\right)=\left[\partial^{2} g\left(x_{i} ; \xi\right) / \partial \xi^{2}\right] \text { and } G_{r}^{\prime \prime}\left(x_{i} ; \xi\right)=\left[\partial^{2} G\left(x_{i} ; \xi\right) / \partial \xi^{2}\right]
$$

\section{Appendix $\mathrm{C}$}

The normal equations are

$$
\begin{aligned}
\frac{\partial \ell_{n}(\boldsymbol{\Theta})}{\partial \beta}= & \frac{n_{0}}{\beta}-\theta \sum_{i=1}^{n_{0}} \log \bar{G}\left(x_{i} ; \xi\right) \\
& -\sum_{i=1}^{n_{2}}\left[\frac{1-\bar{G}\left(r_{i}, \xi\right)^{\theta}}{\bar{G}\left(r_{i}, \xi\right)^{\theta}}\right]^{\beta} \log \left[\frac{1-\bar{G}\left(r_{i}, \xi\right)^{\theta}}{\bar{G}\left(r_{i}, \xi\right)^{\theta}}\right]
\end{aligned}
$$




$$
\begin{aligned}
& +\sum_{i=1}^{n_{0}} \log \left[1-\bar{G}\left(x_{i} ; \xi\right)^{\theta}\right]-\sum_{i=1}^{n_{0}} \frac{\log \left[\frac{1-\bar{G}\left(x_{i} ; \xi\right)^{\theta}}{\bar{G}\left(x_{i} ; \xi\right)^{\theta}}\right]}{\left[\frac{1-\bar{G}\left(x_{i} ; \xi \xi\right)^{\theta}}{\bar{G}\left(x_{i} ; \xi^{\theta}\right.}\right]^{-\beta}} \\
& +\sum_{i=1}^{n_{1}}\left\{\frac{\left[\varphi\left(s_{i}\right)-\varphi\left(s_{i-1}\right)\right]}{\left[\begin{array}{c}
\left(1-\exp \left\{-\left[\frac{1-\bar{G}\left(s_{i}, \xi\right)^{\theta}}{\bar{G}\left(s_{i}, \xi\right)^{\theta}}\right]^{\beta}\right\}\right) \\
-\left(1-\exp \left\{-\left[\frac{1-\bar{G}\left(s_{i-1}, \xi\right)^{\theta}}{\bar{G}\left(s_{i-1}, \xi\right)^{\theta}}\right]^{\beta}\right\}\right)
\end{array}\right]}\right\} .
\end{aligned}
$$

$$
\begin{aligned}
& \frac{\partial \ell_{n}(\boldsymbol{\Theta})}{\partial \theta}=\beta \sum_{i=1}^{n_{2}} \frac{\log \bar{G}\left(r_{i} ; \xi\right)}{\bar{G}\left(r_{i} ; \xi\right)^{\theta}}\left[\frac{1-\bar{G}\left(r_{i}, \xi\right)^{\theta}}{\bar{G}\left(r_{i}, \xi\right)^{\theta}}\right]^{\beta-1} \\
& +\beta \sum_{i=1}^{n_{0}} \frac{\log \bar{G}\left(x_{i} ; \xi\right)}{\bar{G}\left(x_{i} ; \xi\right)^{\theta}\left[\frac{1-\bar{G}\left(x_{i} ; \xi \theta^{\theta}\right.}{\bar{G}\left(x_{i} ; \xi\right)^{\theta}}\right]^{1-\beta}} \\
& +\sum_{i=1}^{n_{1}}\left\{\frac{\left[\psi\left(s_{i}\right)-\psi\left(s_{i-1}\right)\right]}{\left[\begin{array}{c}
\left.\left(1-\exp \left\{-\left[\frac{1-\bar{G}\left(s_{i}, \xi\right)^{\theta}}{\bar{G}\left(s_{i}, \xi\right)^{\theta}}\right]^{\beta}\right\}\right)\right] \\
-\left(1-\exp \left\{-\left[\frac{1-\bar{G}\left(s_{i-1}, \xi\right)^{\theta}}{\bar{G}\left(s_{i-1}, \xi\right)^{\theta}}\right]\right)\right.
\end{array}\right]}\right\}
\end{aligned}
$$




$$
\begin{aligned}
& +\frac{n_{0}}{\theta}-\beta \sum_{i=1}^{n_{0}} \log \bar{G}\left(x_{i} ; \xi\right) \\
& -(\beta-1) \sum_{i=1}^{n_{0}} \frac{\bar{G}\left(x_{i} ; \xi\right)^{\theta} \log \bar{G}\left(x_{i} ; \xi\right)^{\theta}}{\left[1-\bar{G}\left(x_{i} ; \xi\right)^{\theta}\right]}
\end{aligned}
$$

and (for $r=1 \ldots, q$ )

$$
\begin{aligned}
\frac{\partial \ell_{n}(\boldsymbol{\Theta})}{\partial \xi}= & \sum_{i=1}^{n_{0}} \frac{g_{r}^{\prime}\left(x_{i} ; \xi\right)}{g\left(x_{i} ; \xi\right)} \\
& +(\theta \beta+1) \sum_{i=1}^{n_{0}} \frac{G_{r}^{\prime}\left(x_{i} ; \xi\right)}{\bar{G}\left(x_{i} ; \xi\right)} \\
& +\theta(\beta-1) \sum_{i=1}^{n} \frac{G_{r}^{\prime}\left(x_{i} ; \xi\right) \bar{G}\left(x_{i} ; \xi\right)^{\theta-1}}{\left[1-\bar{G}\left(x_{i} ; \xi\right)^{\theta}\right]} \\
& -\beta \theta \sum_{i=1}^{n_{2}} \frac{G_{r}^{\prime}\left(r_{i} ; \xi\right) \bar{G}\left(r_{i} ; \xi\right)^{\theta-1}}{\bar{G}\left(r_{i} ; \xi\right)^{2 \theta}}\left[\frac{1-\bar{G}\left(r_{i}, \xi\right)^{\theta}}{\bar{G}\left(r_{i}, \xi\right)^{\theta}}\right]^{\beta-1} \\
& -\beta \sum_{i=1}^{n_{0}}\left[\frac{1-\bar{G}\left(x_{i} ; \xi\right)^{\theta}}{\bar{G}\left(x_{i} ; \xi\right)^{\theta}}\right]^{\beta-1} \frac{\theta G_{r}^{\prime}\left(x_{i} ; \xi\right) \bar{G}\left(x_{i} ; \xi\right)^{\theta-1}}{\bar{G}\left(x_{i} ; \xi\right)^{2 \theta}} \\
& +\sum_{i=1}^{n_{1}}\left\{\begin{array}{c}
{\left[\zeta\left(s_{i}\right)-\zeta\left(s_{i-1}\right)\right]} \\
\left.\left[\begin{array}{c}
\left(1-\exp \left\{-\left[\frac{1-\bar{G}\left(s_{i}, \xi\right)^{\theta}}{\bar{G}\left(s_{i}, \xi\right)^{\theta}}\right]^{\beta}\right\}\right) \\
-\left(1-\exp \left\{\frac{1-\bar{G}\left(s_{i-1}, \xi\right)^{\theta}}{\bar{G}\left(s_{i-1}, \xi\right)^{\theta}}\right]^{\beta}\right.
\end{array}\right\}\right)
\end{array}\right\}
\end{aligned}
$$


where

$$
\begin{aligned}
& \varphi\left(s_{i}\right)=\exp \left\{-\left[\frac{1-\bar{G}\left(s_{i}, \xi\right)^{\theta}}{\bar{G}\left(s_{i}, \xi\right)^{\theta}}\right]^{\beta}\right\}\left[\frac{1-\bar{G}\left(s_{i}, \xi\right)^{\theta}}{\bar{G}\left(s_{i}, \xi\right)^{\theta}}\right]^{\beta} \log \left[\frac{1-\bar{G}\left(s_{i}, \xi\right)^{\theta}}{\bar{G}\left(s_{i}, \xi\right)^{\theta}}\right] \\
& \varphi\left(s_{i-1}\right)=\exp \left\{-\left[\frac{1-\bar{G}\left(s_{i-1}, \xi\right)^{\theta}}{\bar{G}\left(s_{i-1}, \xi\right)^{\theta}}\right]^{\beta}\right\}\left[\frac{1-\bar{G}\left(s_{i-1}, \xi\right)^{\theta}}{\bar{G}\left(s_{i-1}, \xi\right)^{\theta}}\right]^{\beta} \\
& \times \log \left[\frac{1-\bar{G}\left(s_{i-1}, \xi\right)^{\theta}}{\bar{G}\left(s_{i-1}, \xi\right)^{\theta}}\right], \\
& \psi\left(s_{i}\right)=\frac{-\beta \log \bar{G}\left(s_{i} ; \xi\right)}{\bar{G}\left(s_{i} ; \xi\right)^{\theta}}\left[\frac{1-\bar{G}\left(s_{i}, \xi\right)^{\theta}}{\bar{G}\left(s_{i}, \xi\right)^{\theta}}\right]^{\beta-1} \exp \left\{-\left[\frac{1-\bar{G}\left(s_{i}, \xi\right)^{\theta}}{\bar{G}\left(s_{i}, \xi\right)^{\theta}}\right]^{\beta}\right\} \\
& \psi\left(s_{i-1}\right)=\frac{-\beta \log \bar{G}\left(s_{i-1} ; \xi\right)}{\bar{G}\left(s_{i-1} ; \xi\right)^{\theta}}\left[\frac{1-\bar{G}\left(s_{i-1}, \xi\right)^{\theta}}{\bar{G}\left(s_{i-1}, \xi\right)^{\theta}}\right]^{\beta-1} \\
& \times \exp \left\{-\left[\frac{1-\bar{G}\left(s_{i-1}, \xi\right)^{\theta}}{\bar{G}\left(s_{i-1}, \xi\right)^{\theta}}\right]^{\beta}\right\} \\
& \zeta\left(s_{i}\right)=\beta \theta \frac{G_{r}^{\prime}\left(s_{i} ; \xi\right) \bar{G}\left(s_{i} ; \xi\right)^{\theta-1}}{\bar{G}\left(s_{i} ; \xi\right)^{2 \theta}} \exp \left\{-\left[\frac{1-\bar{G}\left(s_{i}, \xi\right)^{\theta}}{\bar{G}\left(s_{i}, \xi\right)^{\theta}}\right]^{\beta}\right\}\left[\frac{1-\bar{G}\left(s_{i}, \xi\right)^{\theta}}{\bar{G}\left(s_{i}, \xi\right)^{\theta}}\right]^{\beta-1},
\end{aligned}
$$

and

$$
\begin{aligned}
\zeta\left(s_{i-1}\right)= & \beta \theta \frac{G_{r}^{\prime}\left(s_{i-1} ; \xi\right) \bar{G}\left(s_{i-1} ; \xi\right)^{\theta-1}}{\bar{G}\left(s_{i-1} ; \xi\right)^{2 \theta}} \exp \left\{-\left[\frac{1-\bar{G}\left(s_{i-1}, \xi\right)^{\theta}}{\bar{G}\left(s_{i-1}, \xi\right)^{\theta}}\right]^{\beta}\right\} \\
& \times\left[\frac{1-\bar{G}\left(s_{i-1}, \xi\right)^{\theta}}{\bar{G}\left(s_{i-1}, \xi\right)^{\theta}}\right]^{\beta-1} .
\end{aligned}
$$




\section{Appendix D}

Table 4. MLEs obtained using simulated data from WGW density for different parameter settings with $n=200$.

\begin{tabular}{|c|c|c|c|c|c|c|c|c|}
\hline \multicolumn{3}{|c|}{ True } & \multicolumn{3}{|c|}{ MLE } & \multicolumn{3}{|c|}{$\mathrm{SE}$} \\
\hline$\beta$ & $\theta$ & $b$ & $\widehat{\beta}$ & $\widehat{\theta}$ & $\widehat{b}$ & $\widehat{\beta}$ & $\widehat{\theta}$ & $\widehat{b}$ \\
\hline 0.5 & 0.5 & 1 & 0.35 & 0.43 & 1.39 & 0.06 & 0.08 & 0.21 \\
\hline 0.5 & 0.5 & 2 & 0.37 & 0.49 & 2.57 & 0.08 & 0.10 & 0.52 \\
\hline 0.5 & 0.5 & 3 & 0.42 & 0.34 & 3.91 & 0.09 & 0.09 & 0.78 \\
\hline 0.5 & 1 & 1 & 0.35 & 1.14 & 1.39 & 0.06 & 0.11 & 0.21 \\
\hline 0.5 & 1 & 2 & 0.37 & 1.19 & 2.57 & 0.08 & 0.11 & 0.52 \\
\hline 0.5 & 1 & 3 & 0.42 & 0.85 & 3.91 & 0.09 & 0.09 & 0.78 \\
\hline 0.5 & 1.5 & 1 & 0.37 & 1.99 & 1.28 & 0.08 & 0.27 & 0.26 \\
\hline 0.5 & 1.5 & 2 & 0.42 & 1.43 & 2.60 & 0.09 & 0.15 & 0.52 \\
\hline 0.5 & 1.5 & 3 & 0.35 & 1.62 & 4.49 & 0.07 & 0.17 & 0.80 \\
\hline 1 & 0.5 & 1 & 0.56 & 0.38 & 1.80 & 0.12 & 0.07 & 0.36 \\
\hline 1 & 0.5 & 2 & 0.59 & 0.43 & 3.26 & 0.16 & 0.09 & 0.85 \\
\hline 1 & 0.5 & 3 & 0.80 & 0.39 & 4.02 & 0.29 & 0.10 & 1.37 \\
\hline 1 & 1 & 1 & 0.59 & 1.32 & 1.63 & 0.16 & 0.17 & 0.43 \\
\hline 1 & 1 & 2 & 0.80 & 0.98 & 2.68 & 0.29 & 0.09 & 0.91 \\
\hline 1 & 1 & 3 & 0.60 & 1.10 & 5.28 & 0.17 & 0.10 & 1.38 \\
\hline 1 & 1.5 & 1 & 0.59 & 2.56 & 1.63 & 0.16 & 0.74 & 0.43 \\
\hline 1 & 1.5 & 2 & 0.80 & 1.68 & 2.68 & 0.29 & 0.45 & 0.91 \\
\hline 1 & 1.5 & 3 & 0.60 & 2.24 & 5.29 & 0.17 & 0.57 & 1.38 \\
\hline 1.5 & 0.5 & 1 & 0.74 & 0.38 & 1.98 & 0.24 & 0.09 & 0.62 \\
\hline 1.5 & 0.5 & 2 & 1.15 & 0.39 & 2.81 & 0.53 & 0.11 & 1.28 \\
\hline 1.5 & 0.5 & 3 & 0.79 & 0.31 & 6.01 & 0.29 & 0.10 & 2.09 \\
\hline 1.5 & 1 & 1 & 0.74 & 1.49 & 1.98 & 0.24 & 0.30 & 0.62 \\
\hline 1.5 & 1 & 2 & 1.15 & 1.04 & 2.80 & 0.53 & 0.17 & 1.28 \\
\hline 1.5 & 1 & 3 & 0.79 & 1.26 & 6.02 & 0.29 & 0.21 & 2.09 \\
\hline 1.5 & 1.5 & 1 & 1.15 & 1.84 & 1.40 & 0.54 & 0.78 & 0.64 \\
\hline 1.5 & 1.5 & 2 & 0.79 & 2.83 & 4.01 & 0.29 & 1.26 & 1.39 \\
\hline 1.5 & 1.5 & 3 & 1.46 & 1.60 & 3.02 & 0.66 & 0.57 & 1.34 \\
\hline
\end{tabular}


Table 5. MLEs obtained using simulated data from WGW density for different parameter settings with $n=200$.

\begin{tabular}{|c|c|c|c|c|c|c|c|c|}
\hline \multicolumn{3}{|c|}{ True } & \multicolumn{3}{|c|}{ MLE } & \multicolumn{3}{|c|}{$\mathrm{SE}$} \\
\hline$\beta$ & $\theta$ & $b$ & $\widehat{\beta}$ & $\widehat{\theta}$ & $\widehat{b}$ & $\widehat{\beta}$ & $\widehat{\theta}$ & $\widehat{b}$ \\
\hline 0.5 & 0.5 & 1 & 0.62 & 0.53 & 0.84 & 0.15 & 0.07 & 0.19 \\
\hline 0.5 & 0.5 & 2 & 0.53 & 0.57 & 1.81 & 0.10 & 0.06 & 0.31 \\
\hline 0.5 & 0.5 & 3 & 0.48 & 0.52 & 3.01 & 0.08 & 0.06 & 0.45 \\
\hline 0.5 & 1 & 1 & 0.62 & 0.94 & 0.84 & 0.15 & 0.06 & 0.19 \\
\hline 0.5 & 1 & 2 & 0.53 & 1.07 & 1.81 & 0.10 & 0.07 & 0.31 \\
\hline 0.5 & 1 & 3 & 0.48 & 1.05 & 3.01 & 0.08 & 0.07 & 0.45 \\
\hline 0.5 & 1.5 & 1 & 0.53 & 1.54 & 0.90 & 0.10 & 0.16 & 0.15 \\
\hline 0.5 & 1.5 & 2 & 0.48 & 1.58 & 2.00 & 0.08 & 0.16 & 0.30 \\
\hline 0.5 & 1.5 & 3 & 0.57 & 1.36 & 2.83 & 0.13 & 0.15 & 0.61 \\
\hline 1 & 0.5 & 1 & 1.59 & 0.56 & 0.65 & 0.75 & 0.06 & 0.31 \\
\hline 1 & 0.5 & 2 & 1.08 & 0.55 & 1.78 & 0.35 & 0.06 & 0.56 \\
\hline 1 & 0.5 & 3 & 0.89 & 0.49 & 3.27 & 0.23 & 0.06 & 0.79 \\
\hline 1 & 1 & 1 & 1.08 & 1.01 & 0.89 & 0.35 & 0.11 & 0.28 \\
\hline 1 & 1 & 2 & 0.89 & 1.05 & 2.18 & 0.23 & 0.09 & 0.53 \\
\hline 1 & 1 & 3 & 1.29 & 0.91 & 2.46 & 0.52 & 0.09 & 0.98 \\
\hline 1 & 1.5 & 1 & 1.08 & 1.45 & 0.89 & 0.35 & 0.31 & 0.28 \\
\hline 1 & 1.5 & 2 & 0.89 & 1.64 & 2.18 & 0.23 & 0.31 & 0.53 \\
\hline 1 & 1.5 & 3 & 1.29 & 1.28 & 2.46 & 0.52 & 0.29 & 0.98 \\
\hline 1.5 & 0.5 & 1 & 1.68 & 0.54 & 0.85 & 0.79 & 0.07 & 0.40 \\
\hline 1.5 & 0.5 & 2 & 1.25 & 0.48 & 2.35 & 0.42 & 0.07 & 0.77 \\
\hline 1.5 & 0.5 & 3 & 2.04 & 0.53 & 2.31 & 1.11 & 0.08 & 1.27 \\
\hline 1.5 & 1 & 1 & 1.68 & 0.98 & 0.85 & 0.79 & 0.15 & 0.40 \\
\hline 1.5 & 1 & 2 & 1.25 & 1.08 & 2.35 & 0.42 & 0.14 & 0.77 \\
\hline 1.5 & 1 & 3 & 2.04 & 0.91 & 2.31 & 1.11 & 0.12 & 1.26 \\
\hline 1.5 & 1.5 & 1 & 1.25 & 1.73 & 1.17 & 0.42 & 0.50 & 0.39 \\
\hline 1.5 & 1.5 & 2 & 2.04 & 1.24 & 1.54 & 1.11 & 0.38 & 0.84 \\
\hline 1.5 & 1.5 & 3 & 2.30 & 1.13 & 1.96 & 1.52 & 0.35 & 1.31 \\
\hline
\end{tabular}


Table 6. MLEs obtained using simulated data from WG- Li for different parameter settings

\begin{tabular}{|c|c|c|c|c|c|c|}
\hline \multirow[b]{2}{*}{$n$} & \multicolumn{2}{|c|}{ True } & \multicolumn{2}{|c|}{ MLE } & \multicolumn{2}{|c|}{$\mathrm{SE}$} \\
\hline & $\beta$ & $\alpha$ & $\widehat{\beta}$ & $\widehat{\alpha}$ & $\widehat{\beta}$ & $\widehat{\alpha}$ \\
\hline \multirow[t]{9}{*}{200} & 0.5 & 1 & 0.53 & 1.06 & 0.034 & 0.064 \\
\hline & 0.5 & 2 & 0.43 & 2.40 & 0.029 & 0.176 \\
\hline & 0.5 & 3 & 0.48 & 3.11 & 0.032 & 0.222 \\
\hline & 1 & 1 & 0.88 & 1.10 & 0.054 & 0.045 \\
\hline & 1 & 2 & 0.96 & 2.04 & 0.058 & 0.083 \\
\hline & 1 & 3 & 1.10 & 3.12 & 0.066 & 0.118 \\
\hline & 1.5 & 1 & 1.33 & 1.07 & 0.079 & 0.030 \\
\hline & 1.5 & 2 & 1.44 & 2.03 & 0.085 & 0.057 \\
\hline & 1.5 & 3 & 1.66 & 3.08 & 0.097 & 0.080 \\
\hline \multirow[t]{9}{*}{300} & 0.5 & 1 & 0.49 & 1.01 & 0.026 & 0.051 \\
\hline & 0.5 & 2 & 0.55 & 1.86 & 0.029 & 0.094 \\
\hline & 0.5 & 3 & 0.48 & 3.11 & 0.026 & 0.176 \\
\hline & 1 & 1 & 1.07 & 0.97 & 0.051 & 0.027 \\
\hline & 1 & 2 & 0.95 & 2.07 & 0.046 & 0.069 \\
\hline & 1 & 3 & 0.94 & 3.00 & 0.047 & 0.106 \\
\hline & 1.5 & 1 & 1.59 & 0.98 & 0.074 & 0.019 \\
\hline & 1.5 & 2 & 1.43 & 2.05 & 0.067 & 0.048 \\
\hline & 1.5 & 3 & 1.41 & 3.00 & 0.068 & 0.074 \\
\hline
\end{tabular}

\section{Haitham M. Yousof}

Department of Statistics, Mathematics and Insurance,

Benha University,

Benha, Egypt.

email: haitham.yousof@fcom.bu.edu.eg

\section{Mahbubul Majumder}

Department of Mathematics, University of Nebraska, Omaha, NE, USA.

email: mahbub72@gmail.com 


\section{S. M. A. Jahanshahi}

Department of Statistics,

University of Sistan and Baluchestan,

Zahedan, Iran.

email:mjahan@math.usb.ac.ir

\section{G. G. Hamedani}

Department of Mathematics, Statistics

and Computer Science,

Marquette University,

Milwaukee, USA.

email: gholamhoss.hamedani@marquette.edu

\section{Masoom Ali}

Department of Mathematical Sciences, Ball State University,

Muncie, IN, USA.

email: mirmasoom@gmail.com 